\title{
NAMBA FORCING AND NO GOOD SCALE
}

\author{
JOHN KRUEGER
}

\begin{abstract}
We develop a version of Namba forcing which is useful for constructing models with no good scale on $\aleph_{\omega}$. A model is produced in which $\square_{\aleph_{n}}$ holds for all finite $n \geq 1$, but there is no good scale on $\aleph_{\omega}$; this strengthens a theorem of Cummings, Foreman, and Magidor [3] on the non-compactness of square.
\end{abstract}

Cummings, Foreman, and Magidor [4] proved that if the square principle $\square_{\aleph_{n}}$ holds for all finite $n \geq 1$, then there exists a "square-like" sequence on $\aleph_{\omega+1}$ which exhibits uniformity at ordinals of uncountable cofinality. But in [3] they showed that the existence of squares on cardinals less than $\aleph_{\omega}$ fails to imply the existence of a square sequence on $\aleph_{\omega+1}$. Specifically, they constructed a model in which $\square_{\aleph_{n}}$ holds for all $n \geq 1$, but every stationary subset of $\aleph_{\omega+1} \cap \operatorname{cof}\left(\aleph_{0}\right)$ reflects to an ordinal in $\aleph_{\omega+1}$ of cofinality $\aleph_{1}$. Since $\square_{\aleph_{\omega}}$ implies that every stationary subset of $\aleph_{\omega+1}$ contains a non-reflecting stationary subset, $\square_{\aleph_{\omega}}$ fails in this model.

Stationary set reflection is consistent with weaker forms of square. For example, in [2] it is proven that the weak square principle $\square_{\aleph_{\omega}}^{*}$ is consistent with the statement that every family of $\aleph_{1}$ many stationary subsets of $\aleph_{\omega+1} \cap \operatorname{cof}\left(\aleph_{0}\right)$ reflects simultaneously to some ordinal in $\aleph_{\omega+1} \cap \operatorname{cof}\left(\aleph_{1}\right)$. This raises the question whether the square principle holding below $\aleph_{\omega}$ implies any form of weak square principle on $\aleph_{\omega+1}$.

In this paper we rework the forcing construction of [3] to construct a model which satisfies that $\square_{\aleph_{n}}$ holds for all finite $n \geq 1$, but there does not exist a good scale on $\aleph_{\omega}$. Thus the existence of squares below $\aleph_{\omega}$ does not imply even the weakest square principles on $\aleph_{\omega+1}$, such as $\square_{\aleph_{\omega}}^{*}$ and the approachability property $\mathrm{AP}_{\aleph_{\omega}}$. To obtain this model we develop a version of Namba forcing which is useful for constructing models with no good scale.

Section 1 reviews some basic facts about good scales. Sections $2-5$ develop the version of Namba forcing which we will use. In Section 6 we define a forcing iteration of Namba forcing to obtain a model with no good scale on $\aleph_{\omega}$. Section 7 proves the main theorem of the paper; we construct a model which satisfies that $\square_{\aleph_{n}}$ holds for all finite $n \geq 1$, but there is no good scale on $\aleph_{\omega}$.

\section{Good Scales}

The weakest square principle on $\aleph_{\omega+1}$ is the existence of a good scale. In this section we review notation and basic ideas regarding this concept.

For an infinite set $\mathfrak{a} \subseteq \aleph_{0}$, we consider the product $\prod_{n \in \mathfrak{a}} \aleph_{n}$, ordered by eventual domination with respect to $\mathfrak{a}$. For functions $f, g \in \prod_{n \in \mathfrak{a}} \aleph_{n}$, define $f<_{\mathfrak{a}}^{*} g$ if there

2010 Mathematical Subject Classification: 03E35, $03 \mathrm{E} 04$.

Key words and phrases: good scale, Namba forcing. 
exists $n<\omega$ such that for all $k \geq n$ in $\mathfrak{a}, f(k)<g(k)$. The relation $\leq_{\mathfrak{a}}^{*}$ is defined similarly. We also define $f<_{\mathfrak{a}} g$ if for all $n \in \mathfrak{a}, f(n)<g(n)$, and similarly with $\leq_{\mathfrak{a}}$.

A sequence $\left\langle f_{i}: i<\aleph_{\omega+1}\right\rangle$ of functions in $\prod_{n \in \mathfrak{a}} \aleph_{n}$ is a scale if it is $<_{\mathfrak{a}}^{*}$-increasing, and for every function $g$ in $\prod_{n \in \mathfrak{a}} \aleph_{n}$, there is $i<\aleph_{\omega+1}$ such that $g<_{\mathfrak{a}}^{*} f_{i}$.

Any family of $\aleph_{\omega}$ many functions in $\prod_{n \in \mathfrak{a}} \aleph_{n}$ is eventually dominated by some function in $\prod_{n \in \mathfrak{a}} \aleph_{n}$. Thus if $2^{\aleph_{\omega}}=\aleph_{\omega+1}$, then there exists a scale in $\prod_{n \in \mathfrak{a}} \aleph_{n}$. On the other hand, Shelah [11] proved that there always exists an infinite set $\mathfrak{a}$ for which there is a scale in $\prod_{n \in \mathfrak{a}} \aleph_{n}$.

Definition 1.1. Let $\mathfrak{a}$ be an infinite subset of $\aleph_{0}$, and suppose $\left\langle f_{i}: i\left\langle\aleph_{\omega+1}\right\rangle\right.$ is a scale in $\prod_{n \in \mathfrak{a}} \aleph_{n}$. An ordinal $\alpha<\aleph_{\omega+1}$ is said to be good with respect to the scale if there exists a set $A$ cofinal in $\alpha$ and some $n$ such that for any $k \geq n$ in $\mathfrak{a}$, the sequence $\left\langle f_{i}(k): i \in A\right\rangle$ is strictly increasing. The scale is said to be good if there exists a club $C \subseteq \aleph_{\omega+1}$ such that every $\alpha$ in $C$ is good.

Most square-like principles on $\aleph_{\omega+1}$ imply that every scale is good. For example, this is true of the weak square principle $\square_{\aleph_{\omega}}^{*}$ and the approachability property $\mathrm{AP}_{\aleph_{\omega}}$. More generally, if a stationary set $A \subseteq \aleph_{\omega+1}$ is in the approachability ideal $I\left[\aleph_{\omega+1}\right]$, then for any scale, modulo clubs almost every point in $A$ is good with respect to the scale. (See [1].)

Let $\left\langle f_{i}: i<\alpha\right\rangle$ be a $<_{\mathfrak{a}}^{*}$-increasing sequence in $\prod_{n \in \mathfrak{a}} \aleph_{n}$, where $\alpha$ is a limit ordinal. A function $h \in \prod_{n \in \mathfrak{a}} \aleph_{n}$ is an exact upper bound of the sequence if (i) for all $i<\alpha, f_{i}<_{\mathfrak{a}}^{*} h$, and (ii) if $g<_{\mathfrak{a}}^{*} h$ then there exists $i<\alpha$ such that $g<_{\mathfrak{a}}^{*} f_{i}$. It is straightforward to show that any two exact upper bounds of the same sequence are eventually equal.

The only result we will need about good ordinals is the next standard proposition. We include a proof for the convenience of the reader.

Proposition 1.2. Let $\mathfrak{a}$ be an infinite subset of $\aleph_{0}$ and suppose $\left\langle f_{i}: i\left\langle\aleph_{\omega+1}\right\rangle\right.$ is a scale in $\prod_{n \in \mathfrak{a}} \aleph_{n}$. Then for any ordinal $\alpha<\aleph_{\omega+1}$ of uncountable cofinality, the following are equivalent:

(1) $\alpha$ is good with respect to the scale;

(2) for every set $A$ cofinal in $\alpha$, there is a set $B \subseteq A$ cofinal in $\alpha$ and some $n$ such that for all $k \geq n$ in $\mathfrak{a},\left\langle f_{i}(k): i \in B\right\rangle$ is strictly increasing;

(3) there exists an exact upper bound $h$ of the sequence $\left\langle f_{i}: i<\alpha\right\rangle$ such that $\operatorname{cf}(h(n))=\operatorname{cf}(\alpha)$ for all large enough $n \in \mathfrak{a}$.

Proof. $(2 \Rightarrow 1)$ Immediate. $(1 \Rightarrow 2)$ Fix a set $X$ cofinal in $\alpha$ and $n$ such that for all $k \geq n$ in $\mathfrak{a},\left\langle f_{i}(k): i \in X\right\rangle$ is strictly increasing. Let $A$ be cofinal in $\alpha$. Then we can easily define by induction increasing sequences $\left\langle\alpha_{i}: i<\operatorname{cf}(\alpha)\right\rangle$ and $\left\langle\beta_{i}: i<\operatorname{cf}(\alpha)\right\rangle$ of ordinals in $X$ and $A$ respectively such that for all $i, \alpha_{i}<\beta_{i}<\alpha_{i+1}$. For each $i<\operatorname{cf}(\alpha)$, fix $m_{i}<\omega$ such that for all $k \geq m_{i}$ in $\mathfrak{a}, f_{\alpha_{i}}(k)<f_{\beta_{i}}(k)<f_{\alpha_{i+1}}(k)$. As $\operatorname{cf}(\alpha)$ is uncountable, there exists a set $D$ cofinal in $\operatorname{cf}(\alpha)$ and $m$ such that for all $i$ in $D, m_{i}=m$. Let $B=\left\{\beta_{i}: i \in D\right\}$ and let $l=\max \{n, m\}$. Then $B \subseteq A$ is cofinal in $\alpha$. For all $k \geq l$ in $\mathfrak{a}$ and $\beta_{i}<\beta_{j}$ in $B, f_{\beta_{i}}(k)<f_{\alpha_{i+1}}(k) \leq f_{\alpha_{j}}(k)<f_{\beta_{j}}(k)$, where the first and last inequality follow from the fact that $k \geq l \geq m_{i}=m_{j}$, and the second follows from the fact that $k \geq l \geq n$ and $\alpha_{i+1}$ and $\alpha_{j}$ are in $X$.

Let $\operatorname{cf}(\alpha)=\aleph_{m} . \quad(1 \Rightarrow 3)$ Fix $A \subseteq \alpha$ with order type $\aleph_{m}$ and $n>m$ such that for all $k \geq n$ in $\mathfrak{a},\left\langle f_{i}(k): i \in A\right\rangle$ is strictly increasing. For $k \in \mathfrak{a}$, define $h(k)=0$ if $k<n$, and $h(k)=\sup _{i \in A} f_{i}(k)$ if $k \geq n$. Clearly $h$ is an upper bound of 
$\left\langle f_{i}: i<\aleph_{m}\right\rangle$. Suppose $g<_{\mathfrak{a}}^{*} h$. Fix $l>n$ such that for all $k \geq l$ in $\mathfrak{a}, g(k)<h(k)$. Then for each $k \geq l$ in $\mathfrak{a}$, there is $i_{k} \in A$ such that $g(k)<f_{i_{k}}(k)$. Fix $i^{*}>\sup _{k} i_{k}$ in $A$. Then $g<_{\mathfrak{a}}^{*} f_{i^{*}}$.

$(3 \Rightarrow 1)$ Suppose that $h$ is an exact upper bound such that $\operatorname{cf}(h(k))=\aleph_{m}$ for all $k \geq n$ in $\mathfrak{a}$. Choose $\left\langle\gamma_{i}^{k}: i\left\langle\aleph_{m}\right\rangle\right.$ increasing and cofinal in $h(k)$ for all $k \geq n$ in a. For $i<\aleph_{m}$ and $k \in \mathfrak{a}$, define $g_{i}(k)=0$ if $k<n$ and $g_{i}(k)=\gamma_{i}^{k}$ if $k \geq n$. It is straightforward to define by induction increasing sequences $\left\langle\xi_{i}: i<\aleph_{m}\right\rangle$ and $\left\langle\alpha_{i}: i<\aleph_{m}\right\rangle$ cofinal in $\aleph_{m}$ and $\alpha$ respectively such that $g_{\xi_{i}}<_{\mathfrak{a}}^{*} f_{\alpha_{i}}<_{\mathfrak{a}}^{*} g_{\xi_{i+1}}$ for all $i<\aleph_{m}$. Then there is an unbounded set $B \subseteq \aleph_{m}$ and $l$ such that for all $i \in B$ and $k \geq l$ in $\mathfrak{a}, g_{\xi_{i}}(k)<f_{\alpha_{i}}(k)<g_{\xi_{i+1}}(k)$. The set $\left\{\alpha_{i}: i \in B\right\}$ and $l$ witness that $\alpha$ is good. Namely, for $i<j$ in $B$ and $k \geq l$ in $\mathfrak{a}, f_{\alpha_{i}}(k)<g_{\xi_{i+1}}(k) \leq g_{\xi_{j}}(k)<$ $f_{\alpha_{j}}(k)$.

\section{NAMBA FORCING}

A tree is a set of finite sequences, closed under initial segments. The elements of a tree are called nodes of the tree. Note that the union or the intersection of any number of trees is a tree. For finite sequences $\eta$ and $\nu$, we write $\eta \unlhd \nu$ to express that $\eta$ is an initial segment of $\nu$, and $\eta \triangleleft \nu$ to express that $\eta$ is a proper initial segment of $\nu$. We say that $\eta$ and $\nu$ are comparable if $\eta \unlhd \nu$ or $\nu \unlhd \eta$; otherwise they are incomparable. Note that if $\eta$ and $\nu$ are both initial segments of some finite sequence, then they are comparable. A subset of a tree is an antichain if its elements are pairwise incomparable, and is a maximal antichain if it is an antichain and every node of the tree is comparable with one of its elements.

Let $T$ be a tree. We write $\operatorname{Suc}_{T}(\eta)$ for the set $\left\{x: \eta^{\wedge} x \in T\right\}$. A node $\eta$ is a splitting node if $\left|\operatorname{Suc}_{T}(\eta)\right|>1$, and is maximal if $\operatorname{Suc}_{T}(\eta)=\emptyset$. A splitting node $\eta$ is an $n$-splitting node if there are exactly $n$ many splitting nodes of $T$ which are proper initial segments of $\eta$. There is at most one 0 -splitting node of $T$, which if it exists is referred to as the stem of $T$, and denoted by $\operatorname{stem}(T)$. In that case, if $\eta$ is a splitting node of $T$, then $\operatorname{stem}(T) \unlhd \eta$. We let $T_{\eta}$ denote the set of $\nu$ in $T$ which are comparable with $\eta$. Note that $T_{\eta}$ is a tree and is a subset of $T$.

A function $b$ with domain $\omega$ is a branch of $T$ if for all $n, b\lceil n \in T$. The set of branches of $T$ is denoted by $[T]$. An antichain $J \subseteq T$ is a front if every branch of $T$ has an initial segment which is in $J$. The statement " $J$ is a front of $T$ " is absolute between transitive models of set theory. In a tree with no maximal nodes, every front is a maximal antichain. We will mainly be interested in trees with no maximal nodes such that every node can be extended to a splitting node. In such a tree, for every $n<\omega$ the set of $n$-splitting nodes is a front.

Now we define the version of Namba forcing which we will use. Fix a surjection $d: \omega \rightarrow \omega \backslash\{0,1\}$ such that for each $k \geq 2$ : (i) there are infinitely many $n$ such that $d(n)=k$, and (ii) if $n$ is least such that $d(n)=k$, then for all $m<n, d(m)<k$. For example, we can let $d$ be an enumeration of the sequence $2,2,3,2,3,4, \ldots$

Definition 2.1. Let $\mathbb{P}$ be the forcing poset whose conditions are trees $S$ satisfying:

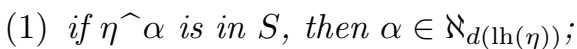

(2) if $\eta$ is a splitting node of $S$, then $\operatorname{Suc}_{S}(\eta)$ is an unbounded subset of $\aleph_{d(\operatorname{lh}(\eta))}$;

(3) for each $\eta$ in $S$ and $k \geq 2$, there is a splitting node $\nu$ of $S$ above $\eta$ such that $d(\operatorname{lh}(\nu))=k$. 
Let $T \leq S$ if $T \subseteq S$. Let $T \leq^{*} S$ if $T \leq S$ and every $\eta$ in $T$ which is a splitting node of $S$ is also a splitting node of $T$.

If $\eta$ is a splitting node of $S$ and $k=d(\operatorname{lh}(\eta))$, then $\operatorname{Suc}_{S}(\eta)$ is an unbounded subset of $\aleph_{k}$, and we say that $\eta$ splits in $\aleph_{k}$.

Note that if $T$ is in $\mathbb{P}$ and $\eta \in T$, then $T_{\eta}$ is in $\mathbb{P}$ and $T_{\eta} \leq T$. If $\eta \in T \leq S$, then $T_{\eta} \leq S_{\eta}$. Also note that if $T \leq S$ then $\operatorname{stem}(S) \unlhd \operatorname{stem}(T)$. It follows that if $S$ and $T$ have incomparable stems, then $S$ and $T$ are incompatible conditions.

Let $S, T \in \mathbb{P}$. For $n<\omega$, define $T \leq_{n} S$ if $T \leq S$ and every $n$-splitting node of $S$ is an $n$-splitting node of $T$. This is equivalent to saying that $T \leq S$ and $T$ and $S$ have the same $n$-splitting nodes. Note that $\leq_{n}$ is a partial ordering, and if $m<n$ then $T \leq_{n} S$ implies $T \leq_{m} S$.

Lemma 2.2. Let $S$ be in $\mathbb{P}$ and $n<\omega$. Let $A$ be the set of nodes of $S$ of the form $\eta \widehat{ } \alpha$, where $\eta$ is an n-splitting node of $S$. Then $A$ has the same cardinality as the set of $n+1$-splitting nodes.

Proof. Every $n+1$-splitting node extends a unique node of the form $\eta \widehat{ } \alpha$, where $\eta$ is an $n$-splitting node of $S$. Conversely, every such node $\eta^{\widehat{\alpha}} \alpha$ extends uniquely to an $n+1$-splitting node.

We will construct conditions using the methods of amalgamation and fusion sequences. Generally speaking, amalgamation is a way to construct a condition by taking the union of a set of subtrees of a condition. Of course, taking such a union will not always produce a condition. The next lemma, whose proof we leave to the interested reader, describes the specific kind of amalgamation we will use.

Lemma 2.3. Let $S$ be in $\mathbb{P}$. Assume that either $B$ is a singleton consisting of a single splitting node of $S$, or $B$ is the set of all n-splitting nodes of $S$ for some $n<\omega$. For each $\eta$ in $B$ let $\operatorname{Suc}(\eta)$ be an unbounded subset of $\operatorname{Suc}_{S}(\eta)$. Let $A=\left\{\eta^{\widehat{\alpha}} \alpha: \eta \in\right.$ $B, \alpha \in \operatorname{Suc}(\eta)\}$. For each $\xi \in A$, let $U(\xi) \leq S_{\xi}$. Then $T=\bigcup\{U(\xi): \xi \in A\}$ is a condition, $T \leq S$, and $\{U(\xi): \xi \in A\}$ is a maximal antichain below $T$. In the case that $B$ is the set of all $n$-splitting nodes of $S, T \leq_{n} S$.

A sequence $\left\langle S_{n}: n<\omega\right\rangle$ of conditions in $\mathbb{P}$ is a fusion sequence if $S_{n+1} \leq_{n} S_{n}$ for all $n$.

Lemma 2.4. Let $\left\langle S_{n}: n<\omega\right\rangle$ be a fusion sequence. For each $n<\omega$, let $J_{n}$ be the set of $n$-splitting nodes of $S_{n}$. Let $T=\bigcap_{n} S_{n}$. Then:

(1) $T=\bigcup_{n}\left\{\xi: \exists \eta \in J_{n} \xi \unlhd \eta\right\}$;

(2) for all $n<\omega, S_{n}$ and $T$ have the same $n$-splitting nodes; so if $T \in \mathbb{P}$, then $T \leq_{n} S_{n}$ for all $n$

(3) $T$ satisfies Definition 2.1(1,2).

The proof is straightforward.

In general, the intersection of a fusion sequence might not be in $\mathbb{P}$, since it could fail Definition 2.1(3). To guarantee that the intersection is a condition, we will construct our fusion sequences carefully, as we now describe.

Notation 2.5. Let $\mathbb{P}_{0}$ be the suborder of $\mathbb{P}$ consisting of conditions $S$ such that for all $n<\omega$, every $n$-splitting node of $S$ splits in $\aleph_{d(n)}$. 
Lemma 2.6. Let $\left\langle S_{n}: n<\omega\right\rangle$ be a fusion sequence such that for all $n<\omega$, every $n$-splitting node of $S_{n}$ splits in $\aleph_{d(n)}$. Then $T=\bigcap_{n} S_{n}$ is in $\mathbb{P}_{0}$.

Proof. By Lemma 2.4(2), if $T$ is in $\mathbb{P}$, then $T$ is in $\mathbb{P}_{0}$. By Lemma 2.4(3), it suffices to verify that $T$ satisfies Definition 2.1(3). Consider $\eta$ in $T$ and let $k \geq 2$. Fix $n>\operatorname{lh}(\eta)$ such that $d(n)=k$. Now $\eta$ is in $S_{n}$, so we can find an $n$-splitting node $\nu$ of $S_{n}$ which is comparable to $\eta$; since $n>\ln (\eta), \eta \unlhd \nu$. Then $\nu$ is an $n$-splitting node of $S_{n}$, so $d(\operatorname{lh}(\nu))=d(n)=k$. By Lemma 2.4(2), $\nu$ splits in $T$.

We describe now in general terms the procedure for defining fusion sequences which we will use in what follows. We start with a condition $S$, and define by induction a fusion sequence $\left\langle S_{n}: n<\omega\right\rangle$. To ensure that the intersection of the sequence is a condition, we arrange that for each $n<\omega$, every $n$-splitting node of $S_{n}$ splits in $\aleph_{d(n)}$.

To begin, choose a node $\eta$ of $S$ which splits in $\aleph_{d(0)}$. Let $S_{0}=S_{\eta}$. Note that the 0-splitting node of $S_{0}$ is $\eta$. Assume that $S_{n}$ is given, and satisfies that every $n$-splitting node of $S_{n}$ splits in $\aleph_{d(n)}$. We would like to define $S_{n+1}$ which has the same $n$-splitting nodes as $S_{n}$, and whose $n+1$-splitting nodes split in $\aleph_{d(n+1)}$. For each $n$-splitting node $\eta$ of $S_{n}$, we can choose a set $\operatorname{Suc}(\eta)$ which is an unbounded subset of $\operatorname{Suc}_{S_{n}}(\eta)$. Let $A_{n}$ be the set of nodes of $S_{n}$ of the form $\eta^{\wedge} \alpha$, where $\eta$ is an $n$-splitting node of $S_{n}$ and $\alpha \in \operatorname{Suc}(\eta)$.

For each $\nu$ in $A_{n}$, we can choose a condition $U(\nu) \leq\left(S_{n}\right)_{\nu}$ whose stem, which extends $\nu$, splits in $\aleph_{d(n+1)}$. Then we let $S_{n+1}=\bigcup\left\{U(\nu): \nu \in A_{n}\right\}$. By Lemma 2.3, $S_{n+1}$ is a condition, $S_{n+1} \leq_{n} S_{n}$, and $\left\{U(\nu): \nu \in A_{n}\right\}$ is a maximal antichain below $S_{n+1}$. Note that the $n+1$-splitting nodes of $S_{n+1}$ are the stems of the conditions $U(\nu)$ for $\nu$ in $A_{n}$, and they split in $\aleph_{d(n+1)}$.

Let $T=\bigcap S_{n}$. Then $T$ is in $\mathbb{P}_{0}$ and satisfies that $T \leq_{n} S_{n}$ for all $n<\omega$. Moreover, for each $n<\omega,\left\{U(\nu): \nu \in A_{n}\right\}$ is a maximal antichain below $T$. Note that the construction of $T$ depends on two kinds of choices, namely, on the successor sets $\operatorname{Suc}(\eta)$ for $n$-splitting nodes $\eta$ of $S_{n}$, and on the conditions $U(\nu)$ for $\nu$ in $A_{n}$.

\section{Properties of Namba Forcing}

In this section we establish some basic properties of the Namba forcing $\mathbb{P}$ introduced in the previous section. The proofs are standard; they rely on Namba forcing type methods which were introduced by Namba [10] and Shelah [12].

Let $\dot{b}$ be a name for $\bigcup \cap \dot{G}$. If $T \leq S$, then $\operatorname{stem}(S) \unlhd \operatorname{stem}(T)$; so if $S$ and $T$ are compatible conditions, then $\operatorname{stem}(S)$ and stem $(T)$ are comparable. It follows that $\bigcap G=\{\xi: \exists S \in \dot{G}(\xi=\operatorname{stem}(S))\}$, and $\dot{b}$ is a function with domain $\omega$ such that for all $n<\omega$, there is $S$ in $G$ such that $\dot{b}\lceil n=\operatorname{stem}(S)$.

Note that $T$ forces that $\operatorname{stem}(T)$ is an initial segment of $\dot{b}$. Hence if $\eta^{-} \alpha \unlhd \operatorname{stem}(T)$, then $T \Vdash \dot{b}(\operatorname{lh}(\eta))=\alpha$.

Lemma 3.1. Suppose $G$ is a generic filter for $\mathbb{P}$, and $S \in G$. Then for all $n<\omega$, $S_{b \nmid n}$ is in $G$.

Proof. It suffices to show that $S_{b\lceil n}$ is comparable with each condition in $G$. So let $T$ be in $G$. Fix $U \leq S, T$ in $G$; by a density argument we can assume that $n<\operatorname{lh}(\operatorname{stem}(U))$. Then $b\left\lceil n \unlhd \operatorname{stem}(U)\right.$, and $U=U_{b \nmid n} \leq S_{b \nmid n}, T$.

Lemma 3.2. For each $k \geq 2, \mathbb{P}$ forces that the set $\{\dot{b}(n): d(n)=k\}$ is unbounded in $\aleph_{k}$. In particular, $\mathbb{P} \Vdash \operatorname{cf}\left(\aleph_{k}\right)=\aleph_{0}$. 
Proof. Let $\alpha<\aleph_{k}$ be given, and let $S \in \mathbb{P}$. We construct a condition $T \leq S$ by applying Lemma 2.3. Fix $\eta$ in $S$ which splits in $\aleph_{k}$; then $d(\operatorname{lh}(\eta))=k$. Let $\operatorname{Suc}(\eta)=\operatorname{Suc}_{S}(\eta) \backslash \alpha$. Let $A=\{\eta \widehat{\beta}: \beta \in \operatorname{Suc}(\eta)\}$. For each $\beta$ in $\operatorname{Suc}(\eta)$, let $U\left(\eta^{\wedge} \beta\right)=S_{\eta \wedge}$. Let $T=\bigcup\{U(\xi): \xi \in A\}$. Then $T \in \mathbb{P}$ and $T \leq S$. Since $U(\eta \widehat{\gamma}) \Vdash \dot{b}(\operatorname{lh}(\eta))=\beta \geq \alpha$ for all $\eta^{\widehat{\beta}} \beta \in A, T \Vdash \dot{b}(\operatorname{lh}(\eta)) \geq \alpha$.

Proposition 3.3. Let $\lambda>\aleph_{\omega}$ be regular. Then $\mathbb{P} \Vdash \operatorname{cf}(\lambda)>\aleph_{0}$.

Proof. Suppose $S$ forces that $\dot{f}: \omega \rightarrow \lambda$ is a function. We define a fusion sequence $\left\langle S_{n}: n<\omega\right\rangle$. Fix $\eta$ in $S$ which splits in $\aleph_{d(0)}$, and let $S_{0}=S_{\eta}$. Assume $S_{n}$ is given. Let $A_{n}$ be the set of nodes of the form $\eta^{\widehat{\alpha}} \alpha$, where $\eta$ is an $n$-splitting node of $S_{n}$ and $\alpha \in \operatorname{Suc}_{S_{n}}(\eta)$. For each $\hat{\eta} \alpha$ in $A_{n}$, choose a condition $V(\eta \widehat{\alpha}) \leq\left(S_{n}\right)_{\eta}{ }^{\alpha} \alpha$ and

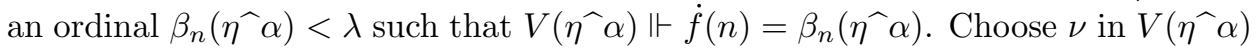
which splits in $\aleph_{d(n+1)}$, and let $U\left(\eta^{\widehat{ }} \alpha\right)=V\left(\eta^{\widehat{ }} \alpha\right)_{\nu}$. Define $S_{n+1}=\bigcup\{U(\xi): \xi \in$ $\left.A_{n}\right\}$.

Let $T=\bigcap_{n} S_{n}$. For each $n, A_{n}$ is a subset of $\aleph_{\omega}^{<\omega}$, and thus has size at most $\aleph_{\omega}$. So if we let $\beta_{n}=\bigcup\left\{\beta_{n}(\xi)+1: \xi \in A_{n}\right\}$, then $\beta_{n}<\lambda$. Now let $\beta=\bigcup_{n} \beta_{n}$, which is less than $\lambda$. We claim that $T \Vdash \dot{f}(n)<\beta$ for all $n$. For suppose that $U \leq T$ decides $\dot{f}(n)$. Then since $\left\{U(\xi): \xi \in A_{n}\right\}$ is a maximal antichain below $T$, for some $\xi$ in $A_{n}, U \Vdash \dot{f}(n)=\beta_{n}(\xi)<\beta$.

Proposition 3.4. The forcing poset $\mathbb{P}$ preserves stationary subsets of $\aleph_{1}$.

Proof. Let $A$ be a stationary subset of $\aleph_{1}$, and we show that $\mathbb{P}$ preserves the stationarity of $A$. So let $S \Vdash \dot{C} \subseteq \aleph_{1}$ is a club, and we find $T \leq S$ and $\delta \in A$ such that $T \Vdash \delta \in \dot{C}$.

For each $\delta<\aleph_{1}$ we define a two-player game $G_{\delta}$ as follows. Let $\eta_{0}$ be the lexicographically least node in $S$ which splits in $\aleph_{d(0)}$. Player I begins the game by choosing $X_{0}$ which is a bounded subset of $\aleph_{d(0)}$, and an ordinal $\beta_{0}<\delta$. Player II responds by choosing an ordinal $\alpha_{0}$ in $\operatorname{Suc}_{S}\left(\eta_{0}\right) \backslash X_{0}$, a condition $U_{0} \leq S_{\eta_{0}}$ - $\alpha_{0}$ whose stem splits in $\aleph_{d(1)}$, and an ordinal $\xi_{0}<\aleph_{1}$ such that $U_{0} \Vdash \xi_{0} \in \dot{C} \backslash \beta_{0}$.

Now suppose $n>0$ and stage $n-1$ of the game is complete, which has resulted in $\eta_{n-1}, X_{n-1}, \beta_{n-1}, \alpha_{n-1}, U_{n-1}$, and $\xi_{n-1}$. Let $\eta_{n}=\operatorname{stem}\left(U_{n-1}\right)$. We assume as an

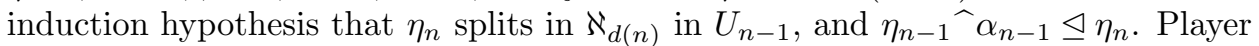
I chooses a set $X_{n}$ which is a bounded subset of $\aleph_{d(n)}$, and an ordinal $\beta_{n}<\delta$. Player II responds by choosing an ordinal $\alpha_{n}$ in $\operatorname{Suc}_{U_{n-1}}\left(\eta_{n}\right) \backslash X_{n}$, a condition $U_{n} \leq\left(U_{n-1}\right)_{\eta_{n}} \alpha_{\alpha_{n}}$ whose stem splits in $\aleph_{d(n+1)}$, and an ordinal $\xi_{n}<\aleph_{1}$ such that $U_{n} \Vdash \xi_{n} \in \dot{C} \backslash \beta_{n}$.

For later purposes, we will refer to the node $\eta_{n}$ described above as the $n$-th node of the partial run of the game. If $n=0$ then the 0 -th node of a partial run is just the lexicographically least node of $S$ which splits in $\aleph_{d(0)}$. If $n>0$ then the $n$-th node is determined after stage $n-1$ of the partial run of the game has completed, and is the stem of the last condition played by Player II and splits in $\aleph_{d(n)}$.

A run of the game produces a sequence of ordinals $\left\langle\xi_{n}: n<\aleph_{0}\right\rangle$ as described above. Player II wins if $\sup _{n} \xi_{n} \leq \delta$. This game is open for Player I. So by the Gale-Stewart Theorem, one of the players has a winning strategy.

We claim that the set of $\delta<\aleph_{1}$ for which Player II has a winning strategy in the game $G_{\delta}$ contains a club. Suppose for a contradiction that there exists a stationary set $Y \subseteq \aleph_{1}$ such that for all $\zeta$ in $Y$, Player I has a winning strategy $\sigma_{\zeta}$ in the game $G_{\zeta}$. Fix a regular cardinal $\theta$ much larger than $\mathbb{P}$ and $\dot{C}$, and let $N$ 
be a countable elementary substructure of $H(\theta)$ such that $N \cap \aleph_{1} \in Y$ and which contains as elements the sets $\mathbb{P}, S, \dot{C}, Y$, and $\left\langle\sigma_{\zeta}: \zeta \in Y\right\rangle$. Let $\delta=N \cap \aleph_{1}$.

We consider simultaneously runs of the games $G_{\zeta}$ for each $\zeta \in Y$, where Player I's moves are according to $G_{\zeta}$ and Player II's moves are in $N$. Suppose it is stage $n$ of the games. For simplicity assume $n>0$; the case $n=0$ is basically the same except with slightly different notation. We assume Player II's previous moves are in $N$ and are the same for all $\zeta$. Then $\eta_{n}$ is in $N$ by elementarity and is the stem of the last condition $U_{n-1}$ played by Player II. For each $\zeta$ in $Y$ apply $\sigma_{\zeta}$ to the moves in the $G_{\zeta}$ run of the game to obtain Player I's next move $\beta_{n}^{\zeta}$ and $X_{n}^{\zeta}$, where $\beta_{n}^{\zeta}<\zeta$ and $X_{n}^{\zeta}$ is a bounded subset of $\aleph_{d(n)}$. Since Player II's moves are in $N$, by elementarity the sequence $\left\langle X_{n}^{\zeta}: \zeta \in Y\right\rangle$ is in $N$. Let $X_{n}=\bigcup_{\zeta} X_{n}^{\zeta}$. Then $X_{n}$ is in $N$ and is a bounded subset of $\aleph_{d(n)}$ since $\aleph_{d(n)}>\aleph_{1}=|Y|$.

Choose some ordinal $\alpha_{n}$ in $\operatorname{Suc}_{U_{n-1}}\left(\eta_{n}\right) \backslash X_{n}$ which is in $N$. Extend $\left(U_{n-1}\right)_{\eta_{n}} \wedge \alpha_{n}$ to a condition $U_{n}$ which decides some $\xi_{n}$ to be in $\dot{C} \backslash \beta_{n}^{\delta}$ and whose stem splits in $\aleph_{d(n+1)}$. By elementarity we can choose $U_{n}$ and $\xi_{n}$ in $N$. Player II now plays $\alpha_{n}$, $U_{n}$, and $\xi_{n}$ as his move in all runs of the game. It might happen that for some $\zeta$ in $Y$, Player II's move violates the rules of the game. However it does not in the game $G_{\delta}$ (and in fact, for stationarily many $\zeta$ ). But we consider the strategies to be total functions so we can continue in any case.

Now note that Player II wins the run of the game $G_{\delta}$, since each $\xi_{n}$ is in $N \cap \aleph_{1}=$ $\delta$. This is a contradiction since Player I used the strategy $\sigma_{\delta}$.

Now we are ready to find $T \leq S$ which forces that $A \cap \dot{C}$ is non-empty. Fix a club $D \subseteq \aleph_{1}$ such that for every $\delta$ in $D$, there is a strategy $\tau_{\delta}$ for Player II in the game $G_{\delta}$. Since $A$ is stationary, we can fix $\delta$ in $D \cap A$. Let $\left\langle\delta_{n}: n<\omega\right\rangle$ be increasing and cofinal in $\delta$.

We define a fusion sequence $\left\langle S_{n}: n<\omega\right\rangle$. Let $\eta_{0}$ be the lexicographically least node in $S$ which splits in $\aleph_{d(0)}$, and let $S_{0}=S_{\eta_{0}}$. Assume now that $S_{n}$ is defined. As an induction hypothesis we assume that every $n$-splitting node $\eta$ of $S_{n}$ is the $n$-th node of a partial run of the game $G_{\delta}$ in which stage $n-1$ has been completed (or $\eta=\eta_{0}$ if $n=0$ ) in which Player II used the strategy $\tau_{\delta}$ and Player I played the ordinals $\delta_{0}, \ldots, \delta_{n-1}$.

Consider an $n$-splitting node $\eta$ of $S_{n}$. If $n=0$ then $\eta=\eta_{0}$ and $\operatorname{Suc}_{S}(\eta)=$ $\operatorname{Suc}_{S_{0}}(\eta)$. If $n>0$ then $\eta$ is the $n$-th node of a partial run of the game with stage $n-1$ having been completed. Let $U_{n-1}$ be the condition played by Player II in the previous move, so that $\eta=\operatorname{stem}\left(U_{n-1}\right)$. As an induction hypothesis assume that $\left(S_{n}\right)_{\eta}=U_{n-1}$. Let $\operatorname{Suc}(\eta)$ be the set of $\alpha$ such that for some move of Player I at stage $n$ whose ordinal part is $\delta_{n}, \tau_{\delta}$ instructs Player II to respond with $\alpha$. So $\operatorname{Suc}(\eta)$ is a subset of $\operatorname{Suc}_{S_{n}}(\eta)$. We claim that $\operatorname{Suc}(\eta)$ is unbounded in $\aleph_{d(n)}$. If not, then Player I can play $\operatorname{Suc}(\eta)$ and $\delta_{n}$, and $\tau_{\delta}$ instructs Player II to play an ordinal in $\operatorname{Suc}(\eta)$ which is a contradiction.

For each $\eta^{\wedge} \alpha$ with $\alpha$ in $\operatorname{Suc}(\eta)$, let $U\left(\eta^{\wedge} \alpha\right) \leq\left(U_{n-1}\right)_{\eta^{\wedge} \alpha}=\left(S_{n}\right)_{\eta^{\wedge} \alpha}$ and $\xi\left(\eta^{\wedge} \alpha\right)$ be the condition and ordinal which $\tau_{\delta}$ instructs Player II to play along with $\alpha$. Let $A_{n}$ be the set of nodes of the form $\eta^{\wedge} \alpha$, where $\eta$ is an $n$-splitting node of $S_{n}$ and $\alpha \in \operatorname{Suc}(\eta)$. Let $S_{n+1}=\bigcup\left\{U(\nu): \nu \in A_{n}\right\}$.

Let $T=\bigcap_{n} S_{n}$. Then $T$ is in $\mathbb{P}$ and $T \leq S$. We claim that $T$ forces $\delta$ is in $\dot{C}$. It suffices to show that for each $n, T$ forces $\dot{C} \cap\left[\delta_{n}, \delta\right]$ is non-empty. But $\left\{U(\nu): \nu \in A_{n}\right\}$ is a maximal antichain below $T$. For each $\nu$ in $A_{n}, U(\nu)$ forces $\xi(\nu)$ is in $\dot{C} \backslash \delta_{n}$. Since $\xi(\nu)$ was part of a move played by Player II according to 
$\tau_{\delta}, \xi(\nu) \leq \delta$. Thus each $U(\nu)$ forces $\dot{C} \cap\left[\delta_{n}, \delta\right]$ is non-empty, and therefore so does $T$.

\section{The Bounding Property}

We now establish the bounding property of the forcing poset $\mathbb{P}$ which will be useful for constructing models with no good scale.

Recall that the surjection $d: \omega \rightarrow \omega \backslash\{0,1\}$, which we used in the definition of $\mathbb{P}$, was chosen to satisfy that whenever $n$ is least such that $d(n)=k$, then $d(m)<k$ for all $m<n$. The next lemma is the reason for this requirement.

Lemma 4.1. Let $k>2$ be given, and suppose that $n$ is least such that $d(n)=k$, so that $n>0$. Let $S$ be in $\mathbb{P}$, and assume that for all $m<n$, every $m$-splitting node of $S$ splits in $\aleph_{d(m)}$. Let $A$ be the set of nodes of $S$ of the form $\eta \widehat{ } \alpha$, where $\eta$ is an $n-1$-splitting node of $S$. Then $|A|<\aleph_{k}$.

Proof. We prove by induction on $m \leq n$ that $S$ has fewer than $\aleph_{k}$ many $m$-splitting nodes. First $S$ has one 0-splitting node. Suppose the claim is true for a fixed $m$ which is less than $n$. Then there are fewer than $\aleph_{k}$ many $m$-splitting nodes of $S$ by the induction hypothesis, and for each $m$-splitting node $\eta,\left|\operatorname{Suc}_{S}(\eta)\right|=\aleph_{d(m)}<\aleph_{k}$, since $d(m)<k$. So the set of nodes in $S$ of the form $\eta \widehat{ } \alpha$, where $\eta$ is an $m$-splitting node, has cardinality less than $\aleph_{k}$. By Lemma 2.2, $S$ has fewer than $\aleph_{k}$ many $m+1$-splitting nodes. Finally, the case $m=n-1$ gives the result.

Proposition 4.2. Every function in $\prod_{n>2} \aleph_{n}^{V}$ in a generic extension by $\mathbb{P}$ is bounded by some function in $\prod_{n \geq 2} \aleph_{n}^{V}$ in the ground model.

Proof. Suppose $S \Vdash \dot{g} \in \prod_{n \geq 2} \aleph_{n}^{V}$. We will define a condition $T \leq S$ and a sequence $\left\langle\zeta_{k}: 2 \leq k<\omega\right\rangle$ such that each $\zeta_{k}$ is in $\aleph_{k}$ and $T \Vdash \dot{g}(k)<\zeta_{k}$. By Lemma 3.2, for each $k \geq 2$ we can fix a name $\dot{m}_{k}$ such that $S \Vdash d\left(\dot{m}_{k}\right)=k \wedge \dot{g}(k)<\dot{b}\left(\dot{m}_{k}\right)$.

We define a fusion sequence $\left\langle S_{n}: n<\omega\right\rangle$. Let $B$ be the set of $n$ such that for some $k \geq 2, n$ is the least integer such that $d(n)=k$. The definition of $S_{n}$ will depend on whether or not $n$ is in $B$. For $n=0, d(0)=2$ and $0 \in B$. Fix $S^{\prime} \leq S$ which decides $\dot{m}_{2}$ as some integer $m_{2}$. Then $d\left(m_{2}\right)=2$. Pick a node $\eta$ of $S^{\prime}$ which splits in $\aleph_{2}$ such that $\operatorname{lh}(\eta)>m_{2}$. Now let $S_{0}=S_{\eta}^{\prime}$. Note that

$$
S_{0} \Vdash \dot{g}(2)<\dot{b}\left(\dot{m}_{2}\right)=\dot{b}\left(m_{2}\right)=\eta\left(m_{2}\right)=\operatorname{stem}\left(S_{0}\right)\left(m_{2}\right) .
$$

Let $\zeta_{2}=\operatorname{stem}\left(S_{0}\right)\left(m_{2}\right)$. Then $S_{0} \Vdash \dot{g}(2)<\zeta_{2}$, and $\zeta_{2}<\aleph_{2}$.

Now suppose that $S_{n}$ is defined. In the first case, assume that $n+1$ is not in $B$. Let $A_{n}$ be the set of nodes of $S_{n}$ of the form $\eta^{\wedge} \alpha$, where $\eta$ is an $n$-splitting node of $S_{n}$. For each $\eta^{\widehat{\alpha}} \alpha$ in $A_{n}$, choose some $\nu$ extending $\eta^{\widehat{\alpha}} \alpha$ which splits in $\aleph_{d(n+1)}$ in $S_{n}$. Let $U(\eta \widehat{\gamma} \alpha)=\left(S_{n}\right)_{\nu}$. Now let $S_{n+1}=\bigcup\left\{U(\xi): \xi \in A_{n}\right\}$.

In the second case, assume that $n+1$ is in $B$. Fix $k$ such that $d(n+1)=k$ and for all $m<n+1, d(m) \neq k$. Let $A_{n}$ be the set of nodes of $S_{n}$ of the form $\eta \widehat{ } \alpha$, where $\eta$ is an $n$-splitting node. Consider $\eta^{\widehat{\alpha}} \alpha$ in $A_{n}$. Extend $\left(S_{n}\right)_{\eta}{ }^{\alpha} \alpha$ to a condition $V(\eta \widehat{\gamma})$ which decides the name $\dot{m}_{k}$ as $m_{k}\left(\eta^{\widehat{\imath}} \alpha\right)$. Then $d\left(m_{k}\left(\eta^{\widehat{\alpha}} \alpha\right)\right)=k$. Now choose a node $\nu$ in $V\left(\eta^{\widehat{\gamma}} \alpha\right)$ which splits in $\aleph_{k}$ and whose length is greater than $m_{k}\left(\eta^{\widehat{ }} \alpha\right)$.

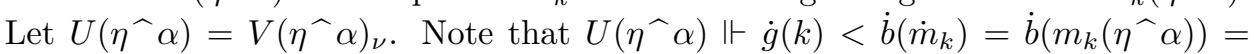
$\nu\left(m_{k}\left(\eta^{\widehat{\alpha}} \alpha\right)\right)=\operatorname{stem}\left(U\left(\eta^{\widehat{\alpha}} \alpha\right)\right)\left(m_{k}\left(\eta^{\widehat{\alpha}} \alpha\right)\right)$. Now let $S_{n+1}=\bigcup\left\{U(\xi): \xi \in A_{n}\right\}$.

By Lemma $4.1, A_{n}$ has size less than $\aleph_{k}$. So letting

$$
\zeta_{k}=\sup \left\{\operatorname{stem}(U(\xi))\left(m_{k}(\xi)\right): \xi \in A_{n}\right\}
$$


$\zeta_{k}<\aleph_{k}$. For each $\xi$ in $A_{n}, U(\xi) \Vdash \dot{g}(k)<\zeta_{k}$. Since $\left\{U(\xi): \xi \in A_{n}\right\}$ is a maximal antichain below $S_{n+1}, S_{n+1} \Vdash \dot{g}(k)<\zeta_{k}$.

This completes the construction of the fusion sequence. Let $T=\bigcap_{n} S_{n}$. Define $h$ in $\prod_{n \geq 2} \aleph_{n}$ by letting $h(k)=\zeta_{k}$. We claim that $T$ forces that $\dot{g}$ is dominated by $h$. So let $k \geq 2$ be given. Let $n$ be the least integer such that $d(n)=k$. As shown above, $S_{n} \Vdash \dot{g}(k)<\zeta_{k}=h(k)$. Hence $T \Vdash \dot{g}(k)<h(k)$.

\section{The $\mathbb{S}$-CONDITION}

We review the $\mathbb{S}$-condition of Shelah [12], and prove that $\mathbb{P}$ satisfies this property. This will ensure that we can iterate $\mathbb{P}$ with revised countable support while not adding reals.

The following concepts are due to Shelah [12].

Definition 5.1. Let $\mathbb{S}$ be a family of regular cardinals larger than $\aleph_{1}$. An $\mathbb{S}$-tree is a pair $(T, H)$, where $T$ is a tree and $H: T \rightarrow \mathbb{S}$ is a function, satisfying:

(1) if $\eta^{\wedge} \alpha$ is in $T$, then $\alpha \in H(\eta)$;

(2) if $\eta$ is a splitting node, then $\operatorname{Suc}_{T}(\eta)$ is an unbounded subset of $H(\eta)$;

(3) for all $\eta$ in $T$, there is a splitting node in $T$ above $\eta$.

Given $\mathbb{I}$-trees $(T, H)$ and $(S, G)$, let $(T, H) \leq(S, G)$ if $T \subseteq S$ and $H=G\lceil T$. Let $(T, H) \leq^{*}(S, G)$ if $T \leq S$ and every $\eta$ in $T$ which is a splitting node of $S$ is also a splitting node of $T$.

The forcing poset $\mathbb{P}$ can be described in the terms just introduced. Let $\mathbb{S}=\left\{\aleph_{n}\right.$ : $2 \leq n<\omega\}$. Define $H:{ }^{<\omega} \aleph_{\omega} \rightarrow \mathbb{S}$ by letting $H(\eta)=\aleph_{d(\operatorname{lh}(\eta))}$. Then a tree $T$ is in $\mathbb{P}$ iff $(T, H\lceil T)$ is an $\mathbb{S}$-tree such that for every $\eta$ in $T$ and $k \geq 2$, there is a splitting node $\nu$ of $T$ above $\eta$ such that $H(\nu)=\aleph_{k}$.

Let $\mathbb{S}$ be a family of regular cardinals larger than $\aleph_{1}$. We define when a forcing poset $\mathbb{Q}$ satisfies the $\mathbb{S}$-condition. This property is described in terms of the existence of a winning strategy for Player II in a certain two-player game. At the end of a run of the game, the players will have constructed an $\mathbb{S}$-tree $(T, H)$ and a function $f: T \rightarrow \mathbb{Q}$, satisfying that $\eta \unlhd \nu$ implies $f(\nu) \leq f(\eta)$.

Suppose it is stage $n$ of the game. If $n>0$, assume that we are given a tree $T_{n}^{I I}$, with no cofinal branches, resulting from the previous play. If $n=0$, then let $T_{0}^{I I}=\{\emptyset\}$. In either case, let $J_{n}^{I I}$ be the set of maximal nodes of $T_{n}^{I I}$. Player I chooses (1) for each $\eta$ in $J_{n}^{I I}$, a finite sequence $\nu$ with $\eta \unlhd \nu$, (2) for each $\xi$ with $\eta \unlhd \xi \triangleleft \nu$, a cardinal $H(\xi) \in \mathbb{S}$ such that $\nu(\operatorname{lh}(\xi)) \in H(\xi)$, and (3) for each $\xi$ with $\eta \triangleleft \xi \unlhd \nu$ (as well as $\xi=\eta=\emptyset$ if $n=0$ ), a condition $f(\xi)$ in $\mathbb{Q}$ such that $\eta \unlhd \zeta \unlhd \xi \unlhd \nu$ implies $f(\xi) \leq f(\zeta)$. This completes Player I's move. Let $J_{n+1}^{I}$ be the set of such finite sequences $\nu$ chosen by Player I, and let $T_{n+1}^{I}$ be the tree $\left\{\xi: \exists \nu \in J_{n+1}^{I} \xi \unlhd \nu\right\}$.

Player II responds by playing, for each $\eta$ in $J_{n+1}^{I}$, (a) a cardinal $H(\eta) \in \mathbb{S}$, (b) a set $\operatorname{Suc}(\eta)$ which is an unbounded subset of $H(\eta)$, and (c) for each $\alpha$ in $\operatorname{Suc}(\eta)$, a condition $f\left(\eta^{\wedge} \alpha\right)$ in $\mathbb{Q}$ such that $f\left(\eta^{\wedge} \alpha\right) \leq f(\eta)$. Let $J_{n+1}^{I I}=\left\{\eta^{\wedge} \alpha: \eta \in J_{n+1}^{I}, \alpha \in\right.$ $\operatorname{Suc}(\eta)\}$, and let $T_{n+1}^{I I}=\left\{\xi: \exists \nu \in J_{n+1}^{I I} \xi \unlhd \nu\right\}$. Note that $J_{n+1}^{I I}$ is the set of maximal nodes of $T_{n+1}^{I I}$.

At the end of the run of the game, an $\mathbb{S}$-tree $(T, H)$ is determined, where $T=$ $\bigcup_{n} T_{n+1}^{I I}$, as well as a function $f: T \rightarrow \mathbb{Q}$ such that $\eta \unlhd \nu$ implies $f(\nu) \leq f(\eta)$. The $n$-splitting nodes of $T$ are exactly the nodes in $J_{n+1}^{I}$, and for each $\eta$ in $J_{n+1}^{I}$, $\operatorname{Suc}_{T}(\eta)=\operatorname{Suc}(\eta)$. 
Player II wins the run of the game if for every $\mathbb{S}$-tree $\left(U, H\lceil U) \leq^{*}(T, H)\right.$, there exists a condition $q \in \mathbb{Q}$ such that

$$
q \Vdash \exists c \in[U] \forall n<\omega(c \nmid n \in \dot{G}) .
$$

We say that $\mathbb{Q}$ satisfies the $\mathbb{S}$-condition if Player II has a winning strategy such that at stage $n$ of a run of the game, for every $\eta$ in $J_{n+1}^{I}$, Player II's choice of $H(\eta)$, $\operatorname{Suc}(\eta)$, and the values $f(\eta \widehat{\alpha})$ for $\alpha \in \operatorname{Suc}(\eta)$, depends only on (i) the node $\eta$, (ii) which initial segments of $\eta$ are in $J_{1}^{I} \cup \cdots \cup J_{n}^{I}$, and (iii) the values of $f$ on the initial segments of $\eta$.

We will use the following theorem.

Theorem 5.2 ([12]). Assume $C H$. Let $\mathbb{Q}$ be a forcing poset which satisfies the $\mathbb{S}$ condition, for some non-empty family $\mathbb{S}$ of regular cardinals larger than $\aleph_{1}$. Then $\mathbb{Q}$ does not add reals.

Let $\mathbb{S}$ be a family of regular cardinals larger than $\aleph_{1}$. If $\mathbb{Q}$ is an $\aleph_{1}$-closed forcing poset, then $\mathbb{Q}$ satisfies the $\mathbb{S}$-condition. In fact, Player II can play any moves which obey the rules of the game. After a run of the game is complete, a tree $(T, H)$ and a function $f: T \rightarrow \mathbb{Q}$ are constructed. If $U \leq^{*} T$, then choose any branch $b$ of $[U]$, and let $q$ be a lower bound of $\{f(b\lceil n): n<\omega\}$. Then $q$ forces $f(b\lceil n)$ is in $\dot{G}$ for all $n<\omega$.

The proof of the next proposition is based on the proof of Shelah [12] that Namba forcing satisfies the $\mathbb{S}$-condition.

Proposition 5.3. Let $\mathbb{S}$ be a family of regular cardinals larger than $\aleph_{1}$ such that for all $2 \leq n<\omega, \aleph_{n}$ is in $\mathbb{S}$. Then $\mathbb{P}$ satisfies the $\mathbb{S}$-condition.

Proof. We describe a winning strategy for Player II. Suppose it is Player II's turn to move at stage $n$ of the game. In particular, we are given a set of nodes $J_{n+1}^{I}$, and values $f(\eta)$ for all $\eta$ in $J_{n+1}^{I}$. Let $\eta$ be in $J_{n+1}^{I}$. Player II's move must be determined only from (i) the node $\eta$, (ii) which initial segments of $\eta$ are in $J_{1}^{I} \cup \cdots \cup J_{n}^{I}$, and (iii) the values of $f$ on the initial segments of $\eta$. Note that the integer $n$ is determined by (i) and (ii).

Let $H(\eta)=\aleph_{d(n)}$. Choose from the Namba condition $f(\eta)$ a node $\nu_{\eta}$ which splits in $\aleph_{d(n)}$. Note that $\operatorname{stem}(f(\eta)) \unlhd \nu_{\eta}$. Let $\operatorname{Suc}(\eta)=\operatorname{Suc}_{f(\eta)}\left(\nu_{\eta}\right)$. For each

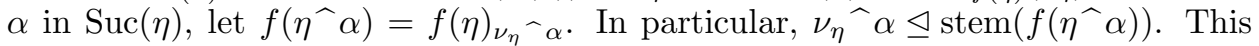
completes the description of Player II's strategy.

We consider a run of the game in which Player II uses the strategy just described. This run of the game produces an $\mathbb{S}$-tree $(T, H)$, a function $f: T \rightarrow \mathbb{P}$, and a mapping $\eta \mapsto \nu_{\eta}$ defined on $\bigcup_{n} J_{n+1}^{I}$.

We claim that for $\eta, \xi \in \bigcup_{n} J_{n+1}^{I}$,

$$
\eta \unlhd \xi \Longleftrightarrow \nu_{\eta} \unlhd \nu_{\xi} .
$$

Let $\eta$ and $\xi$ be given, where $\eta \in J_{n+1}^{I}$ and $\xi \in J_{m+1}^{I}$. Assume $\eta \unlhd \xi$. If $\eta=\xi$, we are done, so assume $\eta \triangleleft \xi$. Then clearly $n<m$. Let $\alpha=\xi(\operatorname{lh}(\eta))$, so that $\eta \widehat{ } \alpha \unlhd \xi$. Then we have

$$
\nu_{\eta} \triangleleft \nu_{\eta} \widehat{\alpha} \unlhd \operatorname{stem}(f(\eta \widehat{ } \alpha)) \unlhd \operatorname{stem}(f(\xi)) \unlhd \nu_{\xi},
$$

where the second relation holds because $f\left(\eta^{\widehat{ }} \alpha\right)=f(\eta)_{\nu_{\eta}}{ }^{\alpha} \alpha$, the third relation holds because $f(\xi) \leq f\left(\eta^{\widehat{\alpha}} \alpha\right)$, and the last relation holds because $\nu_{\xi}$ is a splitting node of $f(\xi)$. Thus $\nu_{\eta} \triangleleft \nu_{\xi}$. 
Conversely, assume $\neg \eta \unlhd \xi$. If $\xi \triangleleft \eta$, then $\nu_{\xi} \triangleleft \nu_{\eta}$ as we just showed; therefore $\neg \nu_{\eta} \unlhd \nu_{\xi}$. So assume that $\eta$ and $\xi$ are incomparable, and we will prove that $\nu_{\eta}$ and $\nu_{\xi}$ are incomparable. Let $k<n$ be the largest integer such that for some $\sigma$ in $J_{k+1}^{I}$, $\sigma \unlhd \eta, \xi$. Note that $k$ exists since the 0 -splitting node of $T$ in $J_{1}^{I}$ is an initial segment of $\eta$ and $\xi$. It follows that there are distinct $\alpha$ and $\beta$ in $\operatorname{Suc}_{T}(\sigma)=\operatorname{Suc}_{f(\sigma)}\left(\nu_{\sigma}\right)$ such that $\sigma^{\wedge} \alpha \unlhd \eta$ and $\sigma^{\wedge} \beta \unlhd \xi$. Then

$$
\nu_{\sigma} \widehat{ } \alpha \unlhd \operatorname{stem}\left(f\left(\sigma^{\widehat{\gamma}} \alpha\right)\right) \unlhd \operatorname{stem}(f(\eta)) \unlhd \nu_{\eta},
$$

so $\nu_{\sigma} \widehat{ } \alpha \unlhd \nu_{\eta}$. Likewise, $\nu_{\sigma} \widehat{\beta} \unlhd \nu_{\xi}$. It follows that $\nu_{\eta}$ and $\nu_{\xi}$ are incomparable. This completes the proof of the claim.

Now we prove that Player II has won the game. Consider $(U, H \uparrow U) \leq^{*}(T, H)$. Note that the $n$-splitting nodes of $U$ are exactly the nodes in $J_{n+1}^{I} \cap U$. We define a fusion sequence $\left\langle S_{n}: n<\omega\right\rangle$ so that the $n$-splitting nodes of $S_{n}$ are exactly the nodes of the form $\nu_{\eta}$, where $\eta$ is in $J_{n+1}^{I} \cap U$, and moreover, they split in $\aleph_{d(n)}$. It follows that $S=\bigcap_{n} S_{n}$ will be a condition.

Let $S_{0}=f(\eta)_{\nu_{\eta}}$, where $\eta$ is the unique node in $J_{1}^{I}=J_{1}^{I} \cap U$. Since $\nu_{\eta}$ splits in $\aleph_{d(0)}$ in $f(\eta), \nu_{\eta}$ is the 0 -splitting node of $S_{0}$ and splits in $\aleph_{d(0)}$. Now assume that $S_{n}$ is defined, so that the $n$-splitting nodes of $S_{n}$ are exactly the nodes of the form $\nu_{\eta}$, where $\eta \in J_{n+1}^{I} \cap U$, and they split in $\aleph_{d(n)}$. Assume moreover that for each $\eta$ in $J_{n+1}^{I} \cap U,\left(S_{n}\right)_{\nu_{\eta}}=f(\eta)_{\nu_{\eta}}$.

We define $S_{n+1}$ by amalgamation. Consider an $n$-splitting node $\nu_{\eta}$ of $S_{n}$, where $\eta$ is in $J_{n+1}^{I} \cap U$. By the choice of Player II's strategy, $\operatorname{Suc}_{T}(\eta)=\operatorname{Suc}_{f(\eta)}\left(\nu_{\eta}\right)=$ $\operatorname{Suc}_{S_{n}}\left(\nu_{\eta}\right)$. Since $U \leq{ }^{*} T, \operatorname{Suc}_{U}(\eta)$ is an unbounded subset of $\operatorname{Suc}_{S_{n}}\left(\nu_{\eta}\right)$. Let $A_{n}$ be the set of nodes of $S_{n}$ of the form $\nu_{\eta} \widehat{\alpha}$, where $\eta \in J_{n+1}^{I} \cap U$ and $\alpha \in \operatorname{Suc}_{U}(\eta)$.

Consider $\nu_{\eta} \widehat{\alpha}$ in $A_{n}$. The node $\eta^{\widehat{\gamma}} \alpha$ is in $U$ and extends uniquely to some $\xi$ in $J_{n+2}^{I} \cap U$. Then $\nu_{\xi}$ is a splitting node of $f(\xi)$. Define $U\left(\nu_{\eta} \widehat{\alpha}\right)=f(\xi)_{\nu_{\xi}}$. Note that $\operatorname{stem}\left(U\left(\nu_{\eta} \widehat{ } \alpha\right)\right)=\nu_{\xi}$. Now by the choice of $f$,

$$
\nu_{\eta} \widehat{\widehat{\alpha}} \unlhd \operatorname{stem}(f(\eta \widehat{ } \alpha)) \unlhd \operatorname{stem}(f(\xi)) \unlhd \nu_{\xi} .
$$

We need to see that $U\left(\nu_{\eta} \widehat{ } \alpha\right) \leq\left(S_{n}\right)_{\nu_{\eta}}{ }^{-} \alpha$. But

$$
U\left(\nu_{\eta} \widehat{ } \alpha\right)=f(\xi)_{\nu_{\xi}} \leq f(\xi)_{\nu_{\eta}{ }^{-} \alpha} \leq f(\eta)_{\nu_{\eta}{ }^{-} \alpha}=\left(S_{n}\right)_{\nu_{\eta}{ }^{-} \alpha}
$$

where the last equality holds since $\left(S_{n}\right)_{\nu_{\eta}}=f(\eta)_{\nu_{\eta}}$. Now let $S_{n+1}=\bigcup\{U(\zeta): \zeta \in$ $\left.A_{n}\right\}$. Then the $n+1$-splitting nodes of $S_{n+1}$ are exactly the stems of the $U(\zeta)$ 's, which are of the form $\nu_{\xi}$ for $\xi \in J_{n+2}^{I} \cap U$, and they split in $\aleph_{d(n+1)}$.

Let $S=\bigcap_{n} S_{n}$. Let $G$ be a generic filter on $\mathbb{P}$ which contains $S$, and let $b=\dot{b}^{G}$. For each $n$, there is an initial segment of $b$ which is an $n$-splitting node of $S$ of the form $\nu_{\eta_{n}}$, for a unique $\eta_{n}$ which is an $n$-splitting node of $U$. Note that for $n<m$, $\nu_{\eta_{n}} \unlhd \nu_{\eta_{m}}$, which implies $\eta_{n} \unlhd \eta_{m}$. Let $c=\bigcup_{n} \eta_{n}$, which is a branch of $U$.

We show that whenever $\xi$ is an initial segment of $c, f(\xi) \in G$. It suffices to show this when $\xi=\eta_{n}$ for some $n$. So consider $\eta_{n}$. Then $\nu_{\eta_{n}}$ is an initial segment of $b$. Also $\nu_{\eta_{n}}$ is an $n$-splitting node of $S_{n}$ and $\left(S_{n}\right)_{\nu_{\eta_{n}}}=f\left(\eta_{n}\right)_{\nu_{\eta_{n}}}$. Now $\nu_{\eta_{n}}$ is in $S$, and $S \leq S_{n}$. So

$$
(S)_{\nu_{\eta_{n}}} \leq\left(S_{n}\right)_{\nu_{\eta_{n}}}=f\left(\eta_{n}\right)_{\nu_{\eta_{n}}} \leq f\left(\eta_{n}\right)
$$

But $\nu_{\eta_{n}}$ is an initial segment of $b$ and $S \in G$, hence $(S)_{\nu_{\eta_{n}}}$ is in $G$ by Lemma 3.1. Therefore $f\left(\eta_{n}\right)$ is in $G$. 


\section{No Good Scales}

We now produce a model by an iteration of Namba forcing in which there is no good scale on $\aleph_{\omega}$. Specifically, the model we produce satisfies that for every infinite set $\mathfrak{a} \subseteq \omega$ and any scale $\left\langle f_{i}: i<\aleph_{\omega+1}\right\rangle$ in $\prod_{n \in \mathfrak{a}} \aleph_{n}$, there are stationarily many $\alpha$ in $\aleph_{\omega+1} \cap \operatorname{cof}\left(\aleph_{1}\right)$ for which there exists an exact upper bound $h$ for $\left\langle f_{i}: i<\alpha\right\rangle$ such that $\operatorname{cf}(h(n))=\aleph_{0}$ for all $n \in \mathfrak{a}$. By Proposition 1.2 and the uniqueness of exact upper bounds, this implies that there is no good scale on $\aleph_{\omega}$.

We construct the model using a revised countable support forcing iteration satisfying the $\mathbb{S}$-condition, for a suitable family of regular cardinals $\mathbb{S}$. This will ensure that no reals are added and GCH holds in the final model. If we did not care about adding reals then we could use the semiproper forcing iteration theorem, as is done in the next section. The iteration theorem we will use is stated next; it comes from [12] and [8].

Theorem 6.1. Let $\left\langle\mathbb{P}_{i}, \dot{\mathbb{Q}}_{j}: i \leq \alpha, j<\alpha\right\rangle$ be a revised countable support forcing iteration, and for each $i<\alpha$, let $\mathbb{S}_{i}$ be a family of regular cardinals larger than $\aleph_{1}$. Suppose that for all $\beta<\alpha$ :

(1) $\mathbb{P}_{\beta}$ forces that $\dot{\mathbb{Q}}_{\beta}$ satisfies the $\mathbb{S}_{\beta}$-condition;

(2) $\mathbb{P}_{\beta}$ forces that every $\xi$ in $\mathbb{S}_{\beta}$ is a regular cardinal greater than or equal to $\aleph_{2}$

(3) (if $\beta$ is not a limit ordinal, or $\operatorname{cf}(\beta)<\beta$, or there is $\gamma<\beta$ with $\left|\mathbb{P}_{\gamma}\right| \geq \beta$ ) there exists $\lambda$ and $\mu$ such that (i) $\lambda^{<\mu}=\lambda$, (ii) if $\xi$ is in $\bigcup\left\{\mathbb{S}_{i}: \beta \leq i<\alpha\right\}$, then $\xi \geq \lambda^{+}$, and (iii) if $\xi$ is in $\bigcup\left\{\mathbb{S}_{j}: j<\beta\right\}$, then $\xi<\mu$;

(4) (if $\operatorname{cf}(\beta)=\beta$ and for all $\gamma<\beta,\left|\mathbb{P}_{\gamma}\right|<\beta$ ) for all $\xi$ in $\mathbb{S}_{\beta}, \xi \geq \beta$.

Then $\mathbb{P}_{\alpha}$ satisfies the $\bigcup\left\{\mathbb{S}_{i}: i<\alpha\right\}$-condition. Moreover, if $\alpha$ is strongly inaccessible and for all $\beta<\alpha,\left|\mathbb{P}_{\beta}\right|<\alpha$, then $\mathbb{P}_{\alpha}$ is $\alpha$-c.c.

We start with a ground model in which GCH holds and $\kappa$ is a supercompact cardinal. We define a revised countable support forcing iteration

$$
\left\langle\mathbb{P}_{i}, \dot{\mathbb{Q}}_{j}: i \leq \kappa, j<\kappa\right\rangle \text {. }
$$

by recursion. We also define families of cardinals $\left\{\mathbb{S}_{i}: i<\kappa\right\}$ satisfying the assumptions of Theorem 6.1. We will maintain that each $\mathbb{S}_{i}$ is countable, and $\left|\mathbb{P}_{i}\right|$ and every cardinal in $\mathbb{S}_{i}$ is less than the least inaccessible greater than $i$.

Let $\mathbb{P}_{0}$ be the trivial forcing. If $\delta \leq \kappa$ is a limit ordinal and $\mathbb{P}_{i}$ is defined for all $i<\delta$, let $\mathbb{P}_{\delta}$ be the revised countable support limit of $\left\langle\mathbb{P}_{i}: i<\delta\right\rangle$. Note that the assumptions of Theorem 6.1 hold for $\mathbb{P}_{\delta}$.

Assume $\mathbb{P}_{\beta}$ is defined, where $\beta<\kappa$. Also assume $\left\{\mathbb{S}_{i}: i<\beta\right\}$ is defined as in Theorem 6.1. Then $\mathbb{P}_{\beta}$ does not add reals, and in particular, it preserves $\aleph_{1}$.

The definition of $\dot{\mathbb{Q}}_{\beta}$ and $\mathbb{S}_{\beta}$ depends on two cases:

Case 1: $\beta$ is not strongly inaccessible.

Case 2: $\beta$ is strongly inaccessible.

First consider Case 1. Let $\dot{\mathbb{Q}}_{\beta}$ be a $\mathbb{P}_{\beta}$-name for $\operatorname{CoLL}\left(\aleph_{1},\left(|\beta|^{+\omega+1}\right)^{V}\right)$, and let $\mathbb{P}_{\beta+1}=\mathbb{P}_{\beta} * \dot{\mathbb{Q}}_{\beta}$. Let $\lambda=\mu$ be the least regular uncountable cardinal larger than $\left|\mathbb{P}_{\beta}\right|$ and all the cardinals in $\bigcup\left\{\mathbb{S}_{i}: i<\beta\right\}$. By GCH, $\lambda^{<\mu}=\lambda$; also $\lambda^{+}$is below the least inaccessible greater than $\beta$. Let $\mathbb{S}_{\beta}=\left\{\lambda^{+}\right\}$. Then the assumptions of Theorem 6.1 hold for $\mathbb{P}_{\beta+1}$. 
Suppose we are in Case 2. Then for all $\gamma<\beta,\left|\mathbb{P}_{\gamma}\right|<\beta$. By Theorem 6.1, $\mathbb{P}_{\beta}$ is $\beta$-c.c. By Case 1 occurring cofinally often below $\beta, \mathbb{P}_{\beta} \Vdash \beta=\aleph_{2}$. Also by induction, $\bigcup\left\{\mathbb{S}_{i}: i<\beta\right\} \subseteq \beta$. Let $\dot{\mathbb{Q}}_{\beta}$ be a $\mathbb{P}_{\beta}$-name for the Namba forcing $\mathbb{P}$ described in the previous sections. Let $\mathbb{S}_{\beta}=\left\{\beta^{+n}: n<\omega\right\}$. The assumptions of Theorem 6.1 hold for $\mathbb{P}_{\beta+1}$.

This completes the definition of $\mathbb{P}_{\kappa}$. It follows by Theorem 6.1 that $\mathbb{P}_{\kappa}$ does not add reals and is $\kappa$-c.c. So $\mathbb{P}_{\kappa}$ collapses $\kappa$ to become $\aleph_{2}$. Also standard arguments show that $\mathbb{P}_{\kappa}$ forces $2^{\mu}=\mu^{+}$for all cardinals $\mu>\aleph_{1}$, so $\mathbb{P}_{\kappa}$ forces GCH.

Let $G$ be a generic filter on $\mathbb{P}_{\kappa}$. We prove that in $V[G]$ there is no good scale on $\aleph_{\omega}$. Note that $\kappa^{+n}=\aleph_{n+2}$ for $n<\omega$, and $\kappa^{+i}=\aleph_{i}$ for $i \geq \omega$. We will abbreviate $\aleph_{i}^{V[G]}$ with $\aleph_{i}$ in what follows.

The first step towards proving that there is no good scale is to extend an elementary embedding. In the ground model $V$, let $j: V \rightarrow M$ be an elementary embedding with critical point $\kappa$ such that $j(\kappa)>\kappa^{+\omega+1}$ and $M$ is closed under $\kappa^{+\omega+1}$-sequences.

Standard arguments using the closure of $M$ show that $j\left(\mathbb{P}_{\kappa}\right)$ factors in $M$ as

$$
j\left(\mathbb{P}_{\kappa}\right)=\mathbb{P}_{\kappa} * \mathbb{P} * \operatorname{CoLL}\left(\aleph_{1},\left(\kappa^{+\omega+1}\right)^{V}\right) * \mathbb{P}_{\kappa+2, j(\kappa)},
$$

where $\mathbb{P}$ is a $\mathbb{P}_{\kappa}$-name for the Namba forcing discussed in the previous sections.

Let $H * I * J$ be a generic filter for $\mathbb{P} * \operatorname{CoLL}\left(\aleph_{1},\left(\kappa^{+\omega+1}\right)^{V}\right) * \mathbb{P}_{\kappa+2, j(\kappa)}$ over $V[G]$. Let $K=G * H * I * J$. Since $j[G]=G \subseteq G * H * I * J=K$, in $V[K]$ we can extend $j$ to $j: V[G] \rightarrow M[K]$ such that $j(G)=K$.

We prove that there is no good scale on $\aleph_{\omega}$ in $V[G]$. Working in this model, fix an infinite set $\mathfrak{a} \subseteq \omega \backslash\{0,1\}$ and suppose $\left\langle f_{i}: i<\aleph_{\omega+1}\right\rangle$ is a scale in $\prod_{n \in \mathfrak{a}} \aleph_{n}$. We will prove that this scale is not good by showing that there are stationarily many $\alpha$ in $\aleph_{\omega+1} \cap \operatorname{cof}\left(\aleph_{1}\right)$ for which there exists an exact upper bound $h$ for $\left\langle f_{i}: i<\alpha\right\rangle$ such that $\operatorname{cf}(h(n))=\aleph_{0}$ for all $n \in \mathfrak{a}$. Fix a club subset $C$ of $\aleph_{\omega+1}$.

Let $j\left(\left\langle f_{i}: i<\aleph_{\omega+1}\right\rangle\right)=\left\langle f_{i}^{j}: i<j\left(\aleph_{\omega+1}\right)\right\rangle$. By the elementarity of $j$, it suffices to show that in $M[K]$ there is an ordinal $\alpha$ in $j(C) \cap \operatorname{cof}\left(\aleph_{1}\right)$ for which there exists an exact upper bound $h$ for $\left\langle f_{i}^{j}: i<\alpha\right\rangle$ such that $\operatorname{cf}(h(n))=\aleph_{0}$ for all $n \in \mathfrak{a}=j(\mathfrak{a})$.

Let $\alpha=\sup \left(j\left[\aleph_{\omega+1}\right]\right)$. We claim that $\alpha$ is in $j(C) \cap \operatorname{cof}\left(\aleph_{1}\right)$ in $M[K]$. By Proposition 3.3, the ordinal $\aleph_{\omega+1}$ is collapsed to have size and cofinality $\aleph_{1}$ by $\mathbb{P} * \operatorname{Colv}\left(\aleph_{1},\left(\kappa^{+\omega+1}\right)^{V}\right)$. Also $\mathbb{P}_{\kappa+2, j(\kappa)}$ preserves $\aleph_{1}$. The set $j\left[\aleph_{\omega+1}\right]$ is in $M$ by the closure of $M$. It follows that $j\left[\aleph_{\omega+1}\right]$ is bounded below $j\left(\aleph_{\omega+1}\right)=\left(\aleph_{\omega+1}\right)^{M[K]}$, and $\alpha$ has cofinality $\aleph_{1}$ in $M[K]$. Since $j[C]$ is a cofinal subset of $\alpha$ contained in $j(C), \alpha$ is in $j(C)$.

Define $h(n)=\sup \left(j\left[\aleph_{n}\right]\right)$ for all $n \in \mathfrak{a}$. Since $\operatorname{cf}\left(\aleph_{n}\right)=\aleph_{0}$ and $j\left[\aleph_{n}\right] \in M[K]$, $\operatorname{cf}(h(n))=\aleph_{0}$ in $M[K]$ and $h(n) \in j\left(\aleph_{n}\right)=\aleph_{n}^{M[K]}$. Hence $h \in \prod_{n \in \mathfrak{a}} \aleph_{n}^{M[K]}$. So it suffices to show that $h$ is an exact upper bound of $\left\langle f_{i}^{j}: i<\alpha\right\rangle$ in $M[K]$.

To see that $h$ is an upper bound, let $i<\alpha$ and we show $f_{i}^{j}<_{\mathfrak{a}}^{*} h$. Since $j\left[\aleph_{\omega+1}\right]$ is cofinal in $\alpha$, fix $\beta<\aleph_{\omega+1}$ such that $i<j(\beta)$. Then $f_{i}^{j}<_{\mathfrak{a}}^{*} f_{j(\beta)}^{j}=j\left(f_{\beta}\right)$. For each $n \in \mathfrak{a}, j\left(f_{\beta}\right)(n)=j\left(f_{\beta}(n)\right) \in j\left[\aleph_{n}\right]$, so $j\left(f_{\beta}\right)(n)<h(n)$. Thus $f_{j(\beta)}^{j}<_{\mathfrak{a}} h$, and therefore $f_{i}^{j}<_{\mathfrak{a}}^{*} h$.

Now let $g$ be a function in $M[K]$ such that $g<_{\mathfrak{a}} h$. Then for all $n \in \mathfrak{a}, g(n)<$ $h(n)=\sup \left(j\left[\aleph_{n}\right]\right)$, so we can fix $\gamma_{n}<\aleph_{n}$ such that $g(n)<j\left(\gamma_{n}\right)$. Let $g_{0}$ be the 
function in $\prod_{n \in \mathfrak{a}} \aleph_{n}$ defined by letting $g_{0}(n)=\gamma_{n}$ for all $n \in \mathfrak{a}$. Then for all $n \in \mathfrak{a}$, $g(n)<j\left(g_{0}(n)\right)$.

The function $g_{0}$ is a countable subset of $\aleph_{\omega}$ in $M[K]=M[G * H * I * J]$, and $\aleph_{\omega}$ has cardinality $\aleph_{1}$ in the model $M[G * H]$. Since $\operatorname{CoLL}\left(\aleph_{1},\left(\kappa^{+\omega+1}\right)^{V}\right) * \mathbb{P}_{\kappa+2, j(\kappa)}$ does not add reals, and thus not countable subsets of $\aleph_{1}, g_{0}$ is in $M[G * H]$. Now $\mathbb{P}$ satisfies the bounding property by Proposition 4.2. So in $M[G]$ there is a function $g_{1}$ in $\prod_{n \in \mathfrak{a}} \aleph_{n}$ such that $g_{0}(n)<g_{1}(n)$ for all $n \in \mathfrak{a}$. Since $\left\langle f_{i}: i<\aleph_{\omega+1}\right\rangle$ is a scale in $M[G]$, we can find $\beta<\aleph_{\omega+1}$ such that $g_{1}<_{\mathfrak{a}}^{*} f_{\beta}$. Fix $n$ such that for all $k \geq n$ in $\mathfrak{a}, g_{1}(k)<f_{\beta}(k)$.

For all $k \in \mathfrak{a}$ greater than or equal to $n$,

$$
g(k)<j\left(g_{0}(k)\right)<j\left(g_{1}(k)\right)<j\left(f_{\beta}(k)\right)=f_{j(\beta)}^{j}(k) .
$$

So $g<_{\mathfrak{a}}^{*} f_{j(\beta)}^{j}$ and $j(\beta)<\alpha$. This proves that $h$ is an exact upper bound as desired.

Remark: Several models have been constructed previously which satisfy the failure of the existence of a good scale on $\aleph_{\omega}$. Foreman and Magidor [6] showed that the Chang's conjecture $\left(\aleph_{\omega+1}, \aleph_{\omega}\right) \rightarrow\left(\aleph_{1}, \aleph_{0}\right)$ implies that there is no good scale on $\aleph_{\omega}$. Magidor [1] showed that the same conclusion follows from Martin's Maximum. Finally, Cummings, Foreman, and Magidor [5] proved that a model of Shelah [9] in which the approachability property $\mathrm{AP}_{\aleph_{\omega}}$ fails also satisfies that there is no good scale.

\section{The Non-Compactness of Square}

We now prove the main result of the paper, and produce a model in which $\square_{\aleph_{n}}$ holds for all $1 \leq n<\omega$, but there is no good scale on $\aleph_{\omega}$.

First we recall the forcing poset from [3] which forces that the square principle $\square_{\aleph_{n}}$ holds for all $1 \leq n<\omega$. This poset is a forcing iteration of length $\omega$ with full support,

$$
\left\langle\mathbb{P}_{n}, \dot{\mathbb{Q}}_{m}: n \leq \omega, m<\omega\right\rangle
$$

where for each $n<\omega, \mathbb{P}_{n}$ forces that $\dot{\mathbb{Q}}_{n}$ is the standard forcing poset for adding

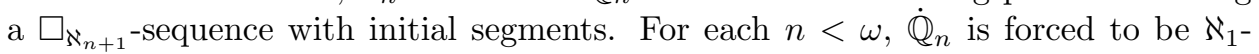
closed and $\left(\aleph_{n+1}\right)+1$-strategically closed. Standard arguments then show that $\mathbb{P}_{\omega}$ is $\aleph_{1}$-closed, and preserves all cardinals and cofinalities. Also, if GCH holds in the ground model, then GCH holds after forcing with $\mathbb{P}_{\omega}$. In what follows we will write $\mathbb{P}(\omega)$ for the iteration $\mathbb{P}_{\omega}$ just described. Note that since $\mathbb{P}(\omega)$ is $\aleph_{1}$-closed, it preserves stationary subsets of $\aleph_{1}$.

We will use the following version of the semiproper forcing iteration theorem $[12]$.

Theorem 7.1. Let $\left\langle\mathbb{P}_{i}, \dot{\mathbb{Q}}_{j}: i \leq \alpha, j<\alpha\right\rangle$ be a revised countable support forcing iteration, where $\alpha$ is a limit ordinal. Assume that for all $i<\alpha, \mathbb{P}_{i}$ forces that $\dot{\mathbb{Q}}_{i}$ is semiproper, and there is $n<\omega$ such that $\mathbb{P}_{i+n} \Vdash\left|\mathbb{P}_{i}\right| \leq \aleph_{1}$. Then $\mathbb{P}_{\alpha}$ is semiproper. Moreover, if $\alpha$ is a Mahlo cardinal and for all $i<\alpha,\left|\mathbb{P}_{i}\right|<\alpha$, then $\mathbb{P}_{\alpha}$ is $\alpha$-c.c.

We will also use Lemma 3 from [7], which states that a certain kind of forcing iteration forces that for any forcing poset $\mathbb{Q}, \mathbb{Q}$ is semiproper iff $\mathbb{Q}$ preserves stationary subsets of $\aleph_{1}$. 
Let $V$ be a model satisfying GCH in which $\kappa$ is a supercompact cardinal. We define in $V$ a revised countable support forcing iteration

$$
\left\langle\mathbb{P}_{i}, \dot{\mathbb{Q}}_{j}: i \leq \kappa, j<\kappa\right\rangle .
$$

Fix a Laver function $l: \kappa \rightarrow V_{\kappa}$.

Let $\mathbb{P}_{0}$ be the trivial forcing. If $\delta \leq \kappa$ is a limit ordinal and $\mathbb{P}_{i}$ is defined for all $i<\delta$, let $\mathbb{P}_{\delta}$ be the revised countable support limit of $\left\langle\mathbb{P}_{i}: i<\delta\right\rangle$.

Assume that $\mathbb{P}_{\alpha}$ is defined. The definition of $\dot{\mathbb{Q}}_{\alpha}$ splits into several cases.

Case A: $\alpha$ is a Mahlo cardinal, and for all $\beta<\alpha,\left|\mathbb{P}_{\beta}\right|<\alpha$.

Case B: not Case A.

If Case B holds, let $\dot{\mathbb{Q}}_{\alpha}$ be a $\mathbb{P}_{\alpha}$-name for the collapse $\operatorname{CoLL}\left(\aleph_{1},\left|\mathbb{P}_{\alpha}\right|^{V}\right)$. Let $\mathbb{P}_{\alpha+1}=\mathbb{P}_{\alpha} * \dot{\mathbb{Q}}_{\alpha}$.

Assume Case A holds. Then $\mathbb{P}_{\alpha}$ is $\alpha$-c.c. and collapses $\alpha$ to become $\aleph_{2}$.

There are three subcases.

Case $\mathrm{A}(1): l(\alpha)$ is a $\mathbb{P}_{\alpha}$-name for a semiproper forcing poset of the form

$$
\mathbb{P}(\omega) * \mathbb{P} * \operatorname{CoLL}\left(\aleph_{1},\left(\alpha^{+\omega+1}\right)^{V}\right) .
$$

Case $\mathrm{A}(2): l(\alpha)$ is a $\mathbb{P}_{\alpha}$-name for a forcing poset of the form $\operatorname{CoLL}\left(\aleph_{1}, \mu\right)$, where $\mu$ is a regular cardinal larger than $\alpha$.

Case $\mathrm{A}(3)$ : Neither $\mathrm{A}(1)$ nor $\mathrm{A}(2)$.

In Case $\mathrm{A}(1)$ and $\mathrm{A}(2)$, we let $\dot{\mathbb{Q}}_{\alpha}$ be the name $l(\alpha)$. In Case $\mathrm{A}(3)$, we let $\dot{\mathbb{Q}}_{\alpha}$ be a $\mathbb{P}_{\alpha}$-name for the collapse $\operatorname{CoLL}\left(\aleph_{1}, \aleph_{2}\right)$.

This completes the definition of $\mathbb{P}_{\kappa}$. Note that $\mathbb{P}_{\kappa}$ is semiproper, $\kappa$-c.c., and collapses $\kappa$ to become $\aleph_{2}$. By Lemma 3 of [7], $\mathbb{P}_{\kappa}$ forces that for every forcing poset $\mathbb{Q}, \mathbb{Q}$ is semiproper iff $\mathbb{Q}$ preserves stationary subsets of $\aleph_{1}$. Since $\mathbb{P}_{\kappa}$ forces that the forcing poset

$$
\mathbb{P}(\omega) * \mathbb{P} * \operatorname{CoLL}\left(\aleph_{1},\left(\kappa^{+\omega+1}\right)^{V}\right)
$$

is a three-step iteration of forcing posets each of which preserves stationary subsets of $\aleph_{1}$, it forces that this poset is semiproper.

Fix an elementary embedding $j: V \rightarrow M$ in the ground model with critical point $\kappa$ such that $M^{\kappa^{+\omega+2}} \subseteq M$ and $j(l)(\kappa)$ is a $\mathbb{P}_{\kappa}$-name for $\mathbb{P}(\omega) * \mathbb{P} *$ $\operatorname{CoLL}\left(\aleph_{1},\left(\kappa^{+\omega+1}\right)^{V}\right)$. Then for any generic filter $G_{\kappa}$ for $\mathbb{P}_{\kappa}, M\left[G_{\kappa}\right]^{\kappa^{+\omega+2}} \subseteq M\left[G_{\kappa}\right]$. So $M\left[G_{\kappa}\right]$ has enough closure to satisfy that the poset $\mathbb{P}(\omega) * \mathbb{P} * \operatorname{CoLL}\left(\aleph_{1},\left(\kappa^{+\omega+1}\right)^{V}\right)$ is semiproper. Therefore by the definition of the forcing iteration, $j\left(\mathbb{P}_{\kappa}\right)$ factors as

$$
j\left(\mathbb{P}_{\kappa}\right)=\mathbb{P}_{\kappa} * \mathbb{P}(\omega) * \mathbb{P} * \operatorname{COLL}\left(\aleph_{1},\left(\kappa^{+\omega+1}\right)^{V}\right) * \mathbb{P}_{\kappa+1, j(\kappa)} .
$$

Let $G_{\kappa} * G(\omega)$ be a generic filter for $\mathbb{P}_{\kappa} * \mathbb{P}(\omega)$ over $V$, and let $W=V\left[G_{\kappa} * G(\omega)\right]$. Then in $W, \kappa=\aleph_{2}$ and $\square_{\aleph_{n}}$ holds for all $1 \leq n<\omega$. We will show that in the model $W$, there is no good scale on $\aleph_{\omega}$.

Note that in $W, \kappa^{+n}=\aleph_{n+2}$ for $n<\omega$ and $\kappa^{+i}=\aleph_{i}$ for $i \geq \omega$. We will abbreviate $\aleph_{i}^{W}$ with $\aleph_{i}$. 
The first step is to generically extend the elementary embedding. This is done exactly as in [3]. Let $H * I * J$ be a generic filter for

$$
\mathbb{P} * \operatorname{CoLL}\left(\aleph_{1},\left(\kappa^{+\omega+1}\right)^{V}\right) * \mathbb{P}_{\kappa+1, j(\kappa)}
$$

over the model $V\left[G_{\kappa}\right][G(\omega)]$. Let $K=G_{\kappa} * G(\omega) * H * I * J$. Since $j\left[G_{\kappa}\right]=G_{\kappa} \subseteq K$, in the model $V[K]$ we can extend $j$ to

$$
j: V\left[G_{\kappa}\right] \rightarrow M[K]
$$

such that $j\left(G_{\kappa}\right)=K$.

Note that in $M\left[G_{\kappa}\right][G(\omega)][H], \aleph_{n}$ has cofinality $\aleph_{0}$ for all $2 \leq n<\omega$. Thus $\operatorname{cf}\left(\aleph_{n}\right)=\aleph_{0}$ in $M[K]$. Also $\operatorname{cf}\left(\aleph_{\omega+1}\right)=\aleph_{1}$ in $M[K]$.

As usual, $M\left[G_{\kappa}\right]^{\kappa^{+\omega+1}} \subseteq M\left[G_{\kappa}\right]$. Also $H\left(\kappa^{+\omega+1}\right)^{V\left[G_{\kappa}\right]}=H\left(\kappa^{+\omega+1}\right)^{V}\left[G_{\kappa}\right]$, and $j\left(\dot{a}^{G_{\kappa}}\right)=j(\dot{a})^{K}$ for any $\overline{\mathbb{P}}_{\kappa}$-name $\dot{a}$ in $V$. Since $j \uparrow H\left(\kappa^{+\omega+1}\right)^{V}$ is in $M$ and $K \in M[K], j\left\lceil H\left(\kappa^{+\omega+1}\right)^{V\left[G_{\kappa}\right]}\right.$ is in $M[K]$. In particular, $j \uparrow G(\omega)$ is in $M[K]$.

In $M[K], j[G(\omega)]$ is a set of conditions in $j(\mathbb{P}(\omega))$. We define a lower bound $q$ of this set by inductively defining $q\lceil n$ for all $n<\omega$. So let $n<\omega$ be given, and assume that $q\lceil n$ is defined and is a lower bound of $\{j(p)\lceil n: p \in G(\omega)\}$.

We claim that

$$
q\left\lceil n \Vdash \bigcup\{\operatorname{dom}(z(n)): z \in j[G(\omega)]\}=\sup \left(j\left[\aleph_{n+2}\right]\right) .\right.
$$

Consider a condition $s$ in $G(\omega)$. Then by genericity we can find $t \leq s$ in $G(\omega)$ such that $t \uparrow n$ decides the value of $\operatorname{dom}(s(n))$, which is some ordinal $\alpha<\aleph_{n+2}$. Then $j(t) \uparrow n$ forces that the domain of $j(s)(n)$ is equal to $j(\alpha)$, and $j(\alpha)<\sup \left(j\left[\aleph_{n+2}\right]\right)$. Conversely if $\gamma<\aleph_{n+2}$, then by genericity there is some $t$ in $G(\omega)$ such that $t \uparrow n$ forces that the domain of $t(n)$ is at least $\gamma$. Then $j(t) \uparrow n$ forces that the domain of $j(t)(n)$ is at least $j(\gamma)$. Since $q \uparrow n \leq j(t) \uparrow n$ for all $t \in G(\omega)$, this proves the claim.

Note that $q\lceil n$ forces that any two conditions in $\{j(p)(n): p \in G(\omega)\}$ are compatible, and hence one is an initial segment of the other by the definition of the ordering on $j\left(\dot{\mathbb{Q}}_{n}\right)$. It follows that $q \uparrow n$ forces that $\bigcup\{j(p)(n): p \in G(\omega)\}$ is a function with domain $\sup \left(j\left[\aleph_{n+2}\right]\right)$. We can find a name $q(n)$ which is forced to extend this function by attaching to the ordinal $\sup \left(j\left[\aleph_{n+2}\right]\right)$ a set of order type $\omega$ which is cofinal in $\sup \left(j\left[\aleph_{n+2}\right]\right)$. Then $q(n)$ is forced to be a condition, since the coherence requirement is trivially satisfied as this set has no limit points. Define $q(n+1)=\left(q\lceil n)^{\wedge} q(n)\right.$.

Now let $L$ be a generic filter on $j(\mathbb{P}(\omega))$ over $V[K]$ which contains $q$. Since $q$ is a lower bound of $j[G(\omega)], j[G(\omega)] \subseteq L$. Hence we can extend $j$ in the model $V[K][L]$ to

$$
j: V\left[G_{\kappa}\right][G(\omega)] \rightarrow M[K][L] .
$$

such that $j\left(G_{\kappa} * G(\omega)\right)=K * L$.

We work in the model $W=V\left[G_{\kappa} * G(\omega)\right]$ and show that there is no good scale on $\aleph_{\omega}$. Let $\mathfrak{a}$ be an infinite subset of $\omega \backslash\{0,1\}$, and suppose that $\left\langle f_{i}: i\left\langle\aleph_{\omega+1}\right\rangle\right.$ is a scale in $\prod_{n \in \mathfrak{a}} \aleph_{n}$. Let $C$ be a club subset of $\aleph_{\omega+1}$. We show that there exists an ordinal in $C \cap \operatorname{cof}\left(\aleph_{1}\right)$ which is not good for the scale.

Let $j\left(\left\langle f_{i}: i<\aleph_{\omega+1}\right\rangle\right)=\left\langle f_{i}^{j}: i<j\left(\aleph_{\omega+1}\right)\right\rangle$. By the elementarity of $j$, it suffices to show that in $M[K][L]$ there is an ordinal in $j(C) \cap \operatorname{cof}\left(\aleph_{1}\right)$ which is not good for the scale $\left\langle f_{i}^{j}: i<j\left(\aleph_{\omega+1}\right)\right\rangle$. 
Let $\alpha=\sup \left(j\left[\aleph_{\omega+1}\right]\right)$. We claim that $\alpha$ is in $j(C) \cap \operatorname{cof}\left(\aleph_{1}\right)$. First note that $\aleph_{\omega+1}$ has size and cofinality $\aleph_{1}$ in the model $M\left[G_{\kappa}\right][G(\omega)][H][I]$. As $\mathbb{P}_{\kappa+1, j(\kappa)} * j(\mathbb{P}(\omega))$ is semiproper over this model, $\aleph_{\omega+1}$ still has size and cofinality $\aleph_{1}$ in $M[K][L]$. Since $j \uparrow \aleph_{\omega+1}$ is in $M, M[K][L]$ models that $\alpha$ has cofinality $\aleph_{1}$. Finally, as $j[C]$ is a cofinal subset of $\alpha$ contained in $j(C), \alpha$ is a limit point of $j(C)$ and hence is in $j(C)$.

In the model $M\left[G_{\kappa}\right][G(\omega)][H][I]$, choose a set $X$ cofinal in $\aleph_{\omega+1}$ with order type $\aleph_{1}$. Also for each $2 \leq n<\omega$, choose a set $S_{n}$ cofinal in $\aleph_{n}$ with order type $\omega$. Since $\mathbb{P}$ satisfies the bounding property and $\operatorname{CoLL}\left(\aleph_{1}, \aleph_{\omega+1}\right)$ is $\aleph_{1}$-closed, every function in $\prod_{n \in \mathfrak{a}} S_{n}$ in $M\left[G_{\kappa}\right][G(\omega)][H][I]$ is bounded by some function in the scale $\left\langle f_{i}: i<\aleph_{\omega+1}\right\rangle$. Conversely, every function in the scale is clearly bounded by some function in $\prod_{n \in \mathfrak{a}} S_{n}$.

It follows that in $M\left[G_{\kappa}\right][G(\omega)][H][I]$ there exists an increasing sequence $\left\langle\beta_{i}: i<\right.$ $\left.\aleph_{1}\right\rangle$ of ordinals in $X$ and a sequence $\left\langle g_{i}: i<\aleph_{1}\right\rangle$ of functions in $\prod_{n \in \mathfrak{a}} S_{n}$ such that for all $i<\aleph_{1}$,

$$
f_{\beta_{i}}<_{\mathfrak{a}} g_{i}<_{\mathfrak{a}}^{*} f_{\beta_{i+1}} .
$$

Suppose for a contradiction that the ordinal $\alpha$ is good in $M[K][L]$. Since $\left\{j\left(\beta_{i}\right)\right.$ : $\left.i<\aleph_{1}\right\}$ is cofinal in $\alpha$, by Proposition 1.2 there is a set $D \subseteq \aleph_{1}$ of size $\aleph_{1}$ and some $n<\omega$ such that for all $k \geq n$ in $\mathfrak{a},\left\langle f_{j\left(\beta_{i}\right)}^{j}(k): i \in D\right\rangle$ is strictly increasing. Now for each $i \in D, f_{j\left(\beta_{i}\right)}^{j}(k)=j\left(f_{\beta_{i}}(k)\right)$. Hence by elementarity, $\left\langle f_{\beta_{i}}(k): i \in D\right\rangle$ is strictly increasing.

We define for each $k \geq n$ in $\mathfrak{a}$ an ordinal $\gamma_{k}$ in $S_{k}$. For each $i \in D$, let $\gamma_{k}^{i}$ be the least ordinal in $S_{k}$ such that $f_{\beta_{i}}(k)<\gamma_{k}^{i}$. Then for $i<j$ in $D, f_{\beta_{i}}(k)<f_{\beta_{j}}(k)$, and therefore $\gamma_{k}^{i} \leq \gamma_{k}^{j}$. But $S_{k}$ has order type $\omega$, so the sequence $\left\langle\gamma_{k}^{i}: i \in D\right\rangle$ is eventually constant. Let $\gamma_{k}$ be the eventual constant value, and let $i_{k}<\aleph_{1}$ be such that $\gamma_{k}^{i}=\gamma_{k}$ for all $i>i_{k}$ in $D$.

Let $i^{*}=\sup \left(\left\{i_{k}+1: k \geq n, k \in \mathfrak{a}\right\}\right)$. Fix $i<j$ in $D$ larger than $i^{*}$. Then

$$
f_{\beta_{i}}<_{\mathfrak{a}} g_{i}<_{\mathfrak{a}}^{*} f_{\beta_{j}} .
$$

Choose some $k \geq 2$ in $\mathfrak{a}$ so that

$$
f_{\beta_{i}}(k)<g_{i}(k)<f_{\beta_{j}}(k) .
$$

Recall that $g_{i}$ is in $\prod_{n \in \mathfrak{a}} S_{n}$. Since $\gamma_{k}$ is the least ordinal in $S_{k}$ such that $f_{\beta_{i}}(k)<\gamma_{k}$, it follows that $\gamma_{k} \leq g_{i}(k)$. But also $f_{\beta_{j}}(k)<\gamma_{k}$, and hence $f_{\beta_{j}}(k)<g_{i}(k)$, which is a contradiction. So indeed $\alpha$ is not good.

Comment: As in [3], the model $W$ satisfies that every stationary subset of $\aleph_{\omega+1} \cap$ $\operatorname{cof}\left(\aleph_{0}\right)$ reflects to an ordinal in $\aleph_{\omega+1} \cap \operatorname{cof}\left(\aleph_{1}\right)$.

\section{REFERENCES}

[1] J. Cummings. Notes on singular cardinal combinatorics. Notre Dame J. Formal Logic, 46(3):251-282, 2005

[2] J. Cummings, M. Foreman, and M. Magidor. Squares, scales, and stationary reflection. J. Math. Log., 1(1):35-98, 2001.

[3] J. Cummings, M. Foreman, and M. Magidor. The non-compactness of square. J. Symbolic Logic, 68(2):637-643, 2003.

[4] J. Cummings, M. Foreman, and M. Magidor. Canonical structure in the universe of set theory. I. Ann. Pure Appl. Logic, 129(1-3):211-243, 2004.

[5] J. Cummings, M. Foreman, and M. Magidor. Canonical structure in the universe of set theory. II. Ann. Pure Appl. Logic, 142(1-3):55-75, 2006. 
[6] M. Foreman and M. Magidor. A very weak square principle. J. Symbolic Logic, 62(1):175-196, 1997.

[7] M. Foreman, M. Magidor, and S. Shelah. Martin's maximum, saturated ideals, and nonregular ultrafilters. I. Ann. of Math. (2), 127(2):1-47, 1988.

[8] M. Gitik and S. Shelah. On the I-condition. Israel J. Math, 48(2-3):148-158, 1984

[9] A. Hajnal, I. Juász, and S. Shelah. Splitting strongly almost disjoint families. Trans. Amer. Math. Soc., 295(1):369-387, 1986.

[10] K. Namba. Independence proof of $\left(\omega, \omega_{\alpha}\right)$-distributivity law in complete Boolean algebras. Comment. Math. Univ. St. Paul., 19:1-12, 1970.

[11] S. Shelah. Cardinal Arithmetic, volume 29 of Oxford Logic Guides. The Clarendon Press, Oxford University Press, New York, second edition, 1994.

[12] S. Shelah. Proper and Improper Forcing. Perspectives in Mathematical Logic. Springer-Verlag, Berlin, second edition, 1998.

Department of Mathematics, University of North Texas

E-mail address: jkrueger@unt.edu 